\title{
ARTICLE
}

\section{Stepwise B-cell-dependent expansion of T helper clonotypes diversifies the $\mathrm{T}$-cell response}

\author{
Julia Merkenschlager ${ }^{1}$, Mickaël J. Ploquin ${ }^{1, \dagger}$, Urszula Eksmond ${ }^{1}$, Rakieb Andargachew ${ }^{2}$, Georgina Thorborn ${ }^{1, \dagger}$,
} Andrew Filby ${ }^{1, \dagger}$, Marion Pepper ${ }^{3}$, Brian Evavold ${ }^{2} \&$ George Kassiotis ${ }^{1,4}$

Antigen receptor diversity underpins adaptive immunity by providing the ground for clonal selection of lymphocytes with the appropriate antigen reactivity. Current models attribute T cell clonal selection during the immune response to T-cell receptor (TCR) affinity for either foreign or self peptides. Here, we report that clonal selection of $\mathrm{CD}^{+}{ }^{+} \mathrm{T}$ cells is also extrinsically regulated by $B$ cells. In response to viral infection, the antigen-specific TCR repertoire is progressively diversified by staggered clonotypic expansion, according to functional avidity, which correlates with self-reactivity. Clonal expansion of lower-avidity T-cell clonotypes depends on availability of MHC II-expressing B cells, in turn influenced by $\mathrm{B}$-cell activation. $\mathrm{B}$ cells clonotypically diversify the $\mathrm{CD} 4{ }^{+} \mathrm{T}$-cell response also to vaccination or tumour challenge, revealing a common effect.

\footnotetext{
${ }^{1}$ Retroviral Immunology, The Francis Crick Institute, Mill Hill Laboratory, London NW7 1AA, UK. ${ }^{2}$ Department of Microbiology and Immunology, Emory University School of Medicine, Atlanta, Georgia 30322, USA. ${ }^{3}$ Department of Immunology, University of Washington, Seattle, Washington 98109-8059, USA. ${ }^{4}$ Department of Medicine, Faculty of Medicine, Imperial College London, London W2 1PG, UK. † Present addresses: Institut Pasteur, Unité HIV Inflammation et Persistance, Paris 75015, France (M.J.P.); William Harvey Research Institute, Queen Mary University of London, London EC1M 6BQ, UK (G.T.); Faculty of Medical Sciences, Newcastle University, Newcastle upon Tyne NE1 7RU, UK (A.F.). Correspondence and requests for materials should be addressed to G.K. (email: george.kassiotis@crick.ac.uk)
} 
A daptive immunity relies on clonal expansion and retention of antigen-specific lymphocytes bearing somatically generated and selected antigen receptors. Selection for lymphocytes with particular T-cell or B-cell receptors (TCRs and BCRs, respectively) occurs throughout the different stages of lymphocyte development, effector response and memory formation, based on thresholds of affinity for self or foreign antigen ${ }^{1-4}$. For example, the strength of TCR signalling is responsible for the selective advantage of high-affinity clonotypes that commonly dominate the peak $\mathrm{CD} 4{ }^{+}$T-cell response ${ }^{5-9}$.

The selective forces driving the dominance of high-affinity $\mathrm{CD}^{+}{ }^{+} \mathrm{T}$ cells during priming may continue to operate during memory formation. However, the relationship between primary expansion of a clonotype and memory formation is not always predictable. Indeed, the potential of distinct TCR clonotypes to form memory following LCMV infection does not correspond to the degree of their primary expansion ${ }^{9}$. Furthermore, comparison of two $\mathrm{CD}^{+}{ }^{+} \mathrm{T}$ clones responding to Listeria monocytogenes infection revealed inverse behaviour during primary and secondary responses ${ }^{10}$, which is linked to avidity for self rather than foreign antigen, setting intrinsic thresholds for sensitivity and responsiveness ${ }^{11}$. The strength of selfreactivity, reflected in the expression levels of CD5, was also proposed as a clonotype-specific property directly related to the strength of TCR signalling in the response of several monoclonal or polyclonal $\mathrm{CD} 4{ }^{+} \mathrm{T}$ cells to foreign antigen ${ }^{12}$. Moreover, individual $\mathrm{CD}^{+} \mathrm{T}$ cells bearing identical TCRs differ considerably in their ability to expand and differentiate in response to $L$. monocytogenes infection ${ }^{13}$, highlighting the influence of stochastic events, not linked to TCR affinity. In addition, the ratio of high- and low-affinity $\mathrm{CD} 4{ }^{+} \mathrm{T}$ cells in response to vaccination is heavily influenced by the co-administered adjuvant ${ }^{14}$, the use of peptide instead of protein antigens ${ }^{15}$, the nature of the vaccine vector ${ }^{16}$ or simply by antigen dose ${ }^{17,18}$. Despite the potential of T-cell-extrinsic factors to influence the clonotypic composition of a T-cell response, their mechanism of action or amplitude are not yet fully understood.

Here, we used a well-characterized model to study the clonotypic evolution of the $\mathrm{CD} 4{ }^{+} \mathrm{T}$-cell response to retroviral infection. Inoculation of C57BL/6 (B6) mice with Friend virus (FV), a retroviral complex of Friend murine leukaemia virus (F-MLV) and spleen focus-forming virus (SFFV), causes protracted infection with several weeks of viral replication ${ }^{19,20}$. We have previously described a TCR $\beta$-transgenic strain, which generates diverse $\mathrm{CD} 4{ }^{+} \mathrm{T}$-cell clonotypes with a range of functional avidities for the dominant $\mathrm{H} 2-\mathrm{A}^{\mathrm{b}}$-restricted env $\mathrm{v}_{122-141}$ epitope within the surface unit of the F-MLV env gene ${ }^{8,21}$. Our results revealed that the $\mathrm{CD} 4{ }^{+} \mathrm{T}$-cell clonotypic hierarchy, set early in the response and determined by TCR avidity, can be reversed later in infection. This pattern of clonotypic progression is created by asynchronous expansion of distinct CD4 ${ }^{+}$T-cell clonotypes, according to antigen reactivity. Importantly, $\mathrm{CD} 4{ }^{+}$ $\mathrm{T}$-cell clonotypic progression relies on $\mathrm{B}$-cell activation and antigen presentation. Thus, not only is the B-cell response to infection helped and clonally diversified by $\mathrm{CD} 4{ }^{+} \mathrm{T}$ cells, it also reciprocally helps and clonally diversifies the $\mathrm{CD}^{+}{ }^{+} \mathrm{T}$-cell response.

\section{Results}

Diversity of virus-specific $\mathrm{CD}^{+}{ }^{+} \mathrm{T}$ cells increases over time. To study the clonotypic composition of an antiviral CD4 ${ }^{+}$T-cell response, we used infection of wild-type (WT) B6 mice with FV. To unequivocally identify a cohort of virus-specific CD4 ${ }^{+} \mathrm{T}$ cells over the course of infection, we used an adoptive transfer system $^{22}$. Mice received allotypically marked EF4.1 T $\mathrm{CR} \beta$-transgenic $\mathrm{CD} 4{ }^{+} \mathrm{T}$ cells $(\sim 10,000$ virus-specific cells engrafted per mouse), at the time of infection. The use of endogenous $\mathrm{TCR} \alpha$ chains in EF4.1 T cells generate a semi-polyclonal TCR repertoire enriched in clonotypes reactive with the F-MLV env ${ }_{122-141}$ epitope $^{21}$. Importantly, pairing of the transgenic TCR $\beta$ chain with TCR V $\alpha 2$ chains (encoded by Trav14 gene segments) or $\mathrm{V} \alpha 3$ chains (encoded by Trav 9 gene segments) creates clonotypes with higher or lower functional avidity, respectively ${ }^{21,23}$.

Numbers of virus-specific donor $\mathrm{CD}^{+} \mathrm{T}$ cells exhibited typical expansion and contraction kinetics (Fig. 1a). As previously observed $^{8}$, the peak response was homogeneously dominated by high-avidity clonotypes, with $\mathrm{V} \alpha 2$ clonotypes rising to $>75 \%$ on day 7 of infection (Fig. 1b). Notably, however, during progression of the response, the frequency of $\mathrm{V} \alpha 2$ clonotypes declined, on average, but varied wildly (5-95\%) between identically treated individual hosts (Fig. 1b).

Although, at the population level, $\mathrm{V} \alpha 2$ clonotypes display higher functional avidity than $\mathrm{V} \alpha 3$ clonotypes, there exists the potential for considerable diversity in the semi-polyclonal repertoire of EF4.1 $\mathrm{T}$ cells ${ }^{21,23}$. To examine whether the observed changes in clonotypic composition were indeed caused by differences in TCR avidity between clonotypes, we measured the frequency of unique TCR clonotypes, by deep-sequencing the endogenous Tcra chains in virus-specific EF4.1 CD4 ${ }^{+} \mathrm{T}$ cells. Using Tcra ${ }^{+/}-\mathrm{EF} 4.1 \mathrm{CD}^{+} \mathrm{T}$ cells, we first confirmed that replacement of $\mathrm{V} \alpha 2$ clonotypes during FV infection was not owing to potential expression of a second TCR $\alpha$ chain (Supplementary Fig. 1). Grouping clones into clonotypes sharing identical amino acid sequences, revealed that the peak (day 7) virus-specific $V \alpha 2$ response was overwhelmingly $(\sim 90 \%)$ dominated by a single clonotype (indicated by red color; CDR3 sequence CAAITGNTGKLIF) in all mice (Fig. 1c). This clonotype was the single largest in the entire virus-specific and non-specific $\mathrm{V} \alpha 2$ preimmune repertoire (Fig. 1c). The frequency of this dominant clonotype remained high $(>60 \%)$ within virus-specific $\mathrm{V} \alpha 2$ cells throughout infection, although it was slightly reduced in comparison with its peak, and additional clonotypes were also visible at later time points (Fig. 1c). In contrast, V $\alpha 3$ cells were considerably more polyclonal (Fig. 1c). The peak of the virus-specific $\mathrm{V} \alpha 3$ response in all mice examined was also dominated by a single clonotype (indicated in red color; CDR3 sequence CVLSGDYSNNRLTL), which however, comprised only $\sim 55 \%$ of $\mathrm{V} \alpha 3$ cells on day 7 and significantly less (between 7 and $46 \%$ in individual mice) at later time points (Fig. 1c). This particular clonotype was rare $(<0.5 \%)$ in the preimmune repertoire (Fig. 1c). Notably, the virus-specific $\mathrm{V} \alpha 3$ response was also characterized by two other groups of clonotypes that exhibited distinct kinetics. The first group comprised of only two 'public' clonotypes (indicated by green colours; CDR3 sequences CALDNTNTGKLTF and CALNVGDNSKLIW), which together made $\sim 10 \%$ of all virus-specific $\mathrm{V} \alpha 3$ cells, a frequency that remained remarkably stable throughout the course of infection (Fig. 1c). The second group comprised of several $(>12)$ distinct clonotypes (indicated by blue-purple colours), all sharing the SNNRIFF motif in their CDR3 sequences, created by the use of the Traj31 segment (Fig. 1c). The latter group contained clonotypes that were not always shared between separate mice, but collectively increased in frequency to $30-50 \%$ of the virus-specific $\mathrm{V} \alpha 3$ cells by day 49 of infection (Fig. 1c). Thus, the clonotypic diversity of virusspecific EF4.1 CD4 ${ }^{+} \mathrm{T}$ cells increased over time owing to the decrease in the proportion of initially dominant $\mathrm{V} \alpha 2$ clonotypes in the overall response, and increase in the representation of Traj31-using clonotypes within the V $\alpha 3$ response. 
a

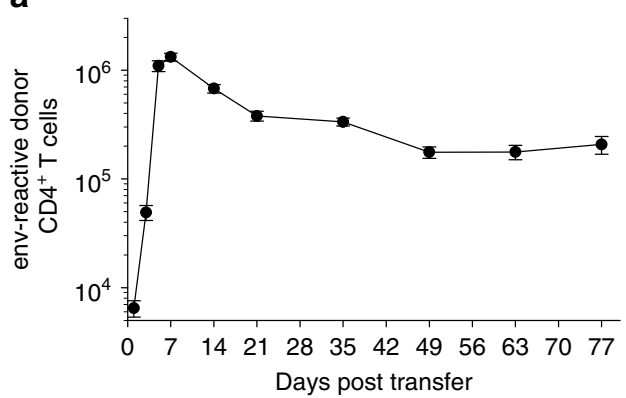

b

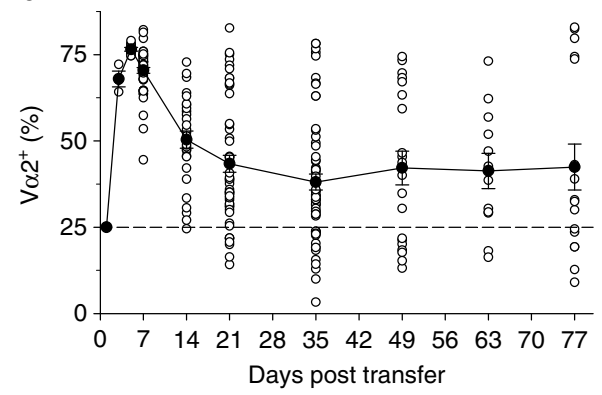

c
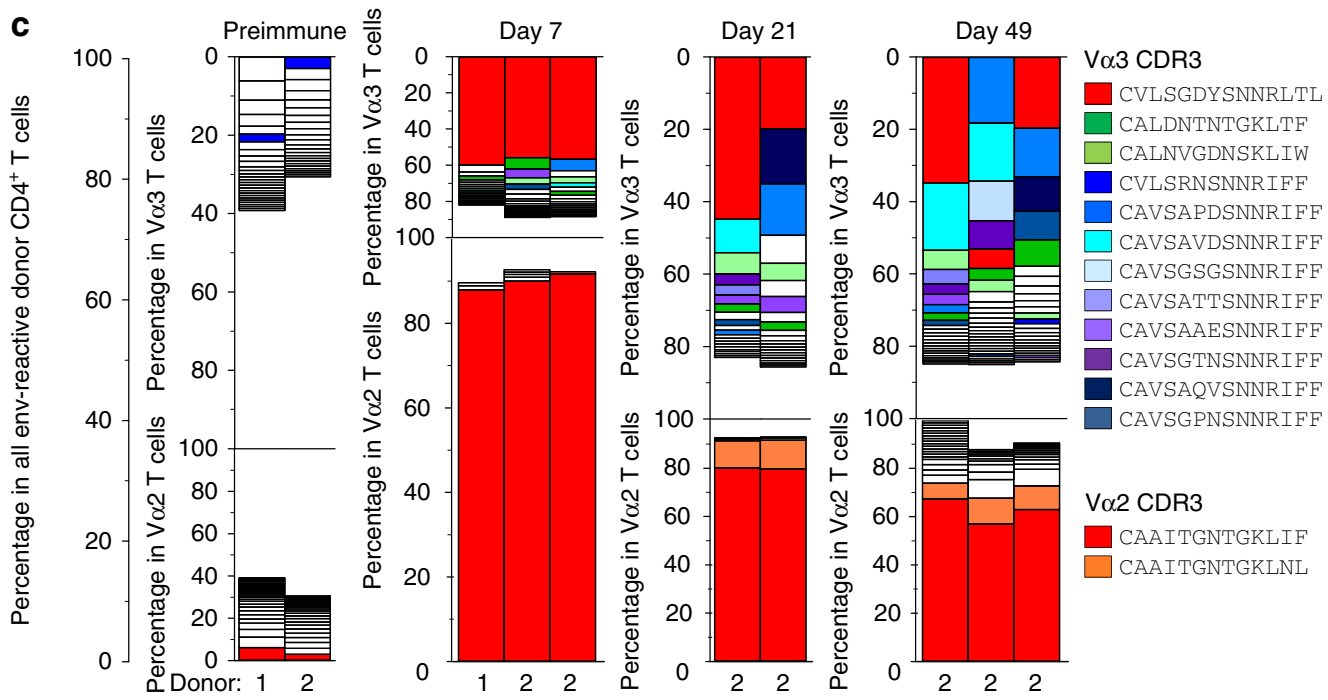

Figure 1 | Clonotypic diversity of virus-specific CD4 ${ }^{+} \mathbf{T}$ cells increases over the course of FV infection. (a) Absolute numbers and (b) $\mathrm{V} \alpha$ composition of env-reactive donor EF4.1 CD4 ${ }^{+}$T cells in the spleens of recipient mice after adoptive T-cell transfer and FV infection ( $n=9-61$ mice per time point; $P<0.001$ between $V \alpha 2$ frequency on day 7 and any later time point, Mann-Whitney rank sum test). (c) Frequency of various $V \alpha 2$ and $V \alpha 3$ clonotypes in total EF4.1 CD4 ${ }^{+}$T cells from two uninfected donor mice (preimmune; donors 1 and 2) or in env-reactive EF4.1 CD4 ${ }^{+}$T cells from the spleens of recipient mice after adoptive T-cell transfer from the indicated donor and FV infection. Each bar graph represents an individual mouse. Clonotypes with frequencies $<0.5 \%$ are not plotted for simplicity. Identical clonotypes within $\mathrm{V} \alpha 2$ and $\mathrm{V} \alpha 3$ subsets are marked with the same color, with red color indicating the dominant $\mathrm{V} \alpha 2$ and $\mathrm{V} \alpha 3$ clonotypes. The scale of the $\mathrm{V} \alpha 2$ and $\mathrm{V} \alpha 3$ plots are adjusted to the relative ratio of the two subsets at a given time point.

Clonotypic behaviour correlates with self-reactivity. Our previous studies indicated lower functional avidity for viral antigen in $\mathrm{V} \alpha 3$ than in $\mathrm{V} \alpha 2$ clonotypes $^{21,23}$, but were limited to the bulk $\mathrm{V} \alpha 2$ and $\mathrm{V} \alpha 3$ populations. To further investigate differences between unique clonotypes using more accurate measures of TCR affinity, we used hybridoma cell lines representing the two major types of behaviour during the response, 'red' $\mathrm{V} \alpha 2$ and Traj31-using 'blue-purple' V $\alpha 3$ (Fig. 2a). These clones were comparably abundant in the preimmune repertoire of EF4.1 mice (Fig. 1c, preimmune) and exemplified the behaviour of other similar clones (Fig. 1c).

The two clones expressed comparable TCR levels (Fig. 2a) and their stimulation with env peptide in vitro reproduced the difference in functional avidity between the bulk $V \propto 2$ and $V \propto 3$ populations (Fig. 2b). Surprisingly, however, the functional avidity of these two clones correlated weakly with binding affinity of the TCR to env pMHC complexes (Fig. 2c). Moreover, precise measurement of two-dimensional TCR affinity for $\mathrm{A}^{\mathrm{b}}$-env $\mathrm{en}_{125-135}$ using a micropipette adhesion frequency assay ${ }^{24,25}$, revealed only minor differences in effective TCR affinities between the two clones (Fig. 2d).

In addition to TCR affinity for antigen, self-reactivity has recently been shown to strongly influence the sensitivity of $\mathrm{CD}^{+}{ }^{+} \mathrm{T}$ cells to antigenic stimulation ${ }^{11,12}$. Therefore, we examined if differences in functional avidity between the selected $\mathrm{V} \alpha 2$ and $\mathrm{V} \alpha 3$ clones reflected differences in selfreactivity. The genes encoding the TCR $\alpha$ chains of these two clones were used to generate two $\mathrm{TCR} \alpha \beta$ doubly transgenic mouse strains (referred to as $\mathrm{EV} \alpha 2$ and $\mathrm{EV} \alpha 3$, respectively) also expressing the same TCR $\beta$ as the TCR $\beta$-transgenic EF4.1 mice. T-cell development proceeded normally in both EV $\alpha 2$ and EV $\alpha 3$ mice, but was considerably more efficient in the former (Fig. 2e,f). Indeed, although total thymocyte numbers were near-normal in both monoclonal TCR-transgenic strains, the frequency and absolute number CD4 single-positive thymocytes was significantly higher in $\mathrm{EV} \alpha 2$ mice (Fig. 2e,f), indicating more efficient positive selection. Consistent with this notion, levels of CD5, which correlate with the strength of self-reactivity, were significantly higher in $\mathrm{EV} \alpha 2$ than in $\mathrm{EV} \alpha 3$ post-selection $\left(\mathrm{CD} 4{ }^{+} \mathrm{CD} 8{ }^{-} \mathrm{TCR}^{\text {high }}\right.$ ) thymocytes (Fig. $\left.2 \mathrm{~g}\right)$. In comparison with cells from the parental EF4.1 strain, which expressed normal CD5 levels (Supplementary Fig. 2a), EV $\alpha 2$ and EV $\alpha 3$ thymocytes expressed 63 and 34\%, respectively, of normal CD5 levels. Peripheral $\mathrm{CD}^{+}{ }^{+} \mathrm{T}$-cell numbers, which also reflect the degree of self-reactivity ${ }^{26}$, were comparable to the WT levels in EV $\alpha 2$ mice, but significantly reduced in EV $\alpha 3$ mice (Supplementary Fig. 2b). Mature $\mathrm{CD} 4^{+} \mathrm{T}$ cells were homogenously naive $\left(\mathrm{CD} 44^{\text {low }} \mathrm{CD} 25^{-}\right)$in both monoclonal TCR-transgenic strains and expressed comparable TCR levels (Supplementary Fig. 2c). CD5 levels were reduced in both EV $\alpha 2$ 
a
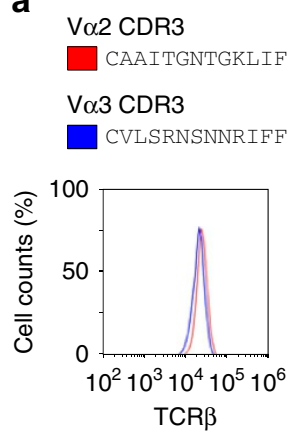

b

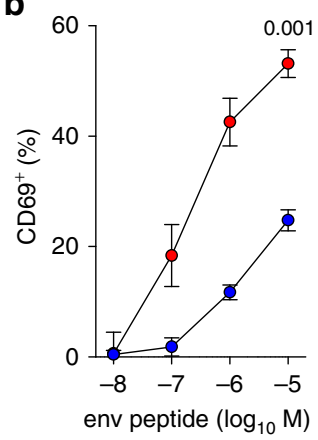

c

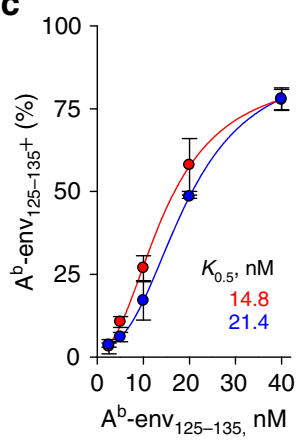

d

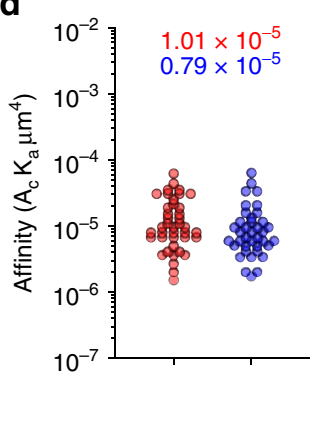

e

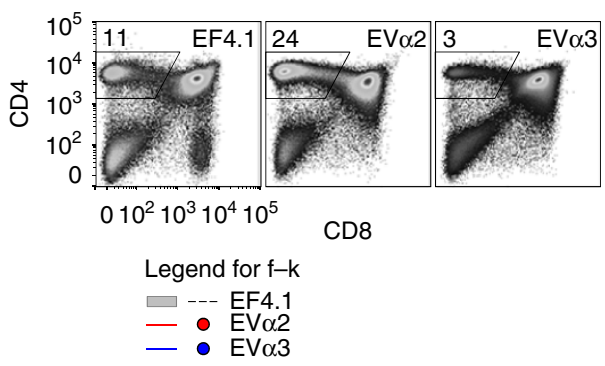

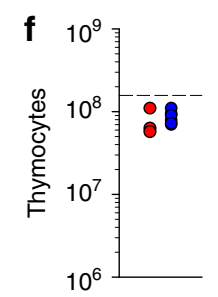

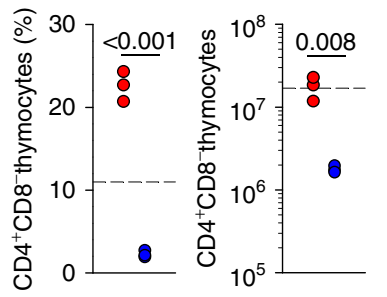

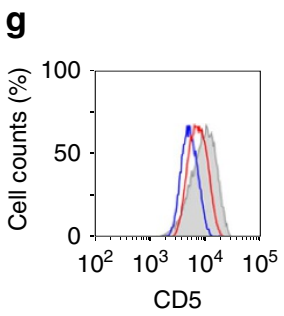

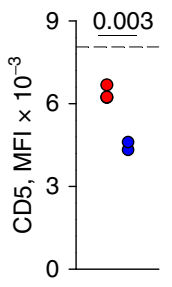

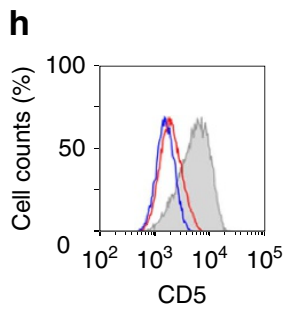

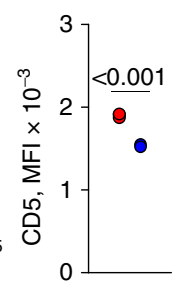

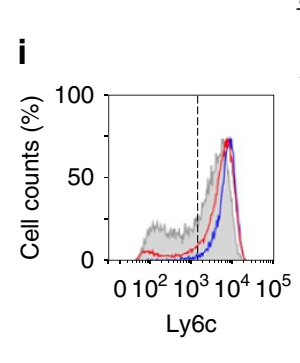

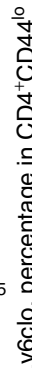
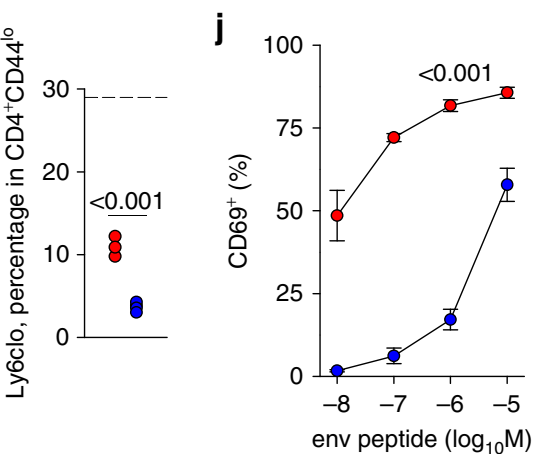

$\mathbf{k}$

k $10^{-2}=3.25 \times 10^{-5}$

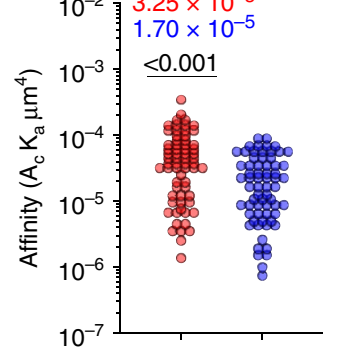

Figure 2 | Development and reactivity with self and foreign antigen of monoclonal EV $\boldsymbol{\alpha} \mathbf{2}$ and $\mathbf{E V} \boldsymbol{\alpha} \mathbf{3} \mathbf{T}$ cells. (a) CDR3 sequences and TCR $\beta$ expression levels in two hybridoma cells lines representing the indicated clonotypes. (b) Responsiveness of the same hybridoma cells lines as in a to overnight stimulation with env $122-141$ peptide, measured by CD69 upregulation $(n=2)$. (c) Frequency of $A^{b}$-env $v_{125-135}$ tetramer-positive cells, at different tetramer concentration, following staining of the same hybridoma cells lines as in a and normalized for TCR expression. Symbols represent the means ( \pm s.e.m., $n=2$ ) of experimental data, whereas lines are fits using a four-parameter Hill equation. Numbers next to the regression lines denote apparent affinity $\left(K_{0.5}\right)$ estimates. (d) Effective two-dimensional TCR affinities for $A^{b}$-env $125-135$ of T-cell hybridomas measured by the micropipette adhesion frequency assay and normalized by TCR surface density. Each individual data point represents the affinity of a single T cell. Numbers in the plot represent the effective affinity geometric mean of the population. (e) Flow cytometric analysis of thymocyte development in EF4.1, EV $\alpha 2$ and EV $\alpha 3$ TCR-transgenic mice. (f) Absolute number of total thymocytes and frequency and number of CD4 single-positive thymocytes in the same mice as in e. (g) CD5 levels in post-selection ( $C D 4{ }^{+} \mathrm{CD} 8-{ }^{-} \mathrm{TCR}^{\text {high }}$ ) thymocytes from the same mice as in e. (h) CD5 levels in naive splenic CD4 ${ }^{+} \mathrm{T}$ cells from EV $\alpha 2$ and $\mathrm{EV} \alpha 3$ mice. (i) Ly6c levels in naive splenic CD4 ${ }^{+} \mathrm{T}$ cells from the same mice. In $\mathbf{f}-\mathbf{i}$, each symbol represents an individual mouse from one representative of two experiments. Horizontal dashed lines represent the mean values for control EF4.1 mice. (j) Responsiveness of EV $\alpha 2$ and EV $\alpha 3$ T cells to overnight env $_{124-138}$ peptide stimulation. Responses were measured by CD69 upregulation and are plotted as means $( \pm$ s.e.m., $n=3-4)$. (k) Effective two-dimensional TCR affinities for $A^{b}$-env ${ }_{125-135}$ of primary $T$ cells measured by the micropipette adhesion frequency assay and normalized by TCR surface density, and plotted as described in $\mathbf{d}$.

and $\mathrm{EV} \alpha 3$ peripheral $\mathrm{T}$ cell in comparison with thymocytes and with WT T cells (Fig. 2h). This was likely because of the relative paucity of MHC II-expressing cells in the periphery of these B-cell-deficient Rag1 ${ }^{-1-} \mathrm{EV} \alpha 2$ and $\mathrm{EV} \alpha 3$ mice. Indeed, comparison of EV $\alpha 2 \mathrm{~T}$ cells from B-cell-deficient Rag1 ${ }^{-1-}$ or B-cell-sufficient Rag1 ${ }^{+1+}$ mice revealed a specific role for B cells in maintaining CD5 levels in peripheral, but not thymic $\mathrm{CD}^{+}{ }^{+} \mathrm{T}$ cells (Supplementary Fig. 2d,e). Nevertheless, CD5 levels remained significantly higher in EV $\alpha 2$ than in $\mathrm{EV} \alpha 3$ peripheral T cells (Fig. 2h) and loss of Ly6C expression, which signifies higher self-reactivity ${ }^{27}$, marked a higher proportion of
$\mathrm{EV} \alpha 2$ than in $\mathrm{EV} \alpha 3$ peripheral $\mathrm{T}$ cells (Fig. 2i). Phenotypic differences between monoclonal $\mathrm{EV} \alpha 2$ and $\mathrm{EV} \alpha 3 \mathrm{~T}$ cells correlated remarkably well with functional avidity, which was reflected in their sensitivity to in vitro env peptide stimulation (Fig. 2j). In contrast, two-dimensional TCR affinity for $\mathrm{A}^{\mathrm{b}}$-env $125-135$ differed only by a factor of two between the two clones (Fig. 2k). Although this difference was statistically significant, it was too small to account for the disparity in antigen reactivity. Thus, functional avidity of $\mathrm{EV} \alpha 2$ and $\mathrm{EV} \alpha 3$ $\mathrm{T}$ cells better correlated with self-reactivity than with affinity for antigen. 
T-cell clonal composition depends on infection kinetics. In B6 mice, acute FV infection is followed by chronic low-level infection, which can be ultimately cleared with kinetics that likely differ most significantly between FV-infected hosts. An effect of viral load on clonotypic composition was suggested by a strong correlation between F-MLV DNA copy numbers in the spleens of $\mathrm{FV}$-infected recipients and frequency of $\mathrm{V} \alpha 2$ clonotypes in virusspecific $\mathrm{CD} 4^{+} \mathrm{T}$ cells 35 days post infection (Fig. 3a). However, this positive correlation (Fig. 3a) argued against relative loss of $\mathrm{V} \alpha 2$ clonotypes owing to excessive activation, as suggested by studies in another TCR-transgenic system ${ }^{10,11}$. To examine whether viral load directly determined the clonotypic composition of the antiviral T-cell response, we infected adult B6 mice with N-tropic F-MLV (F-MLV-N), which is restricted by

a
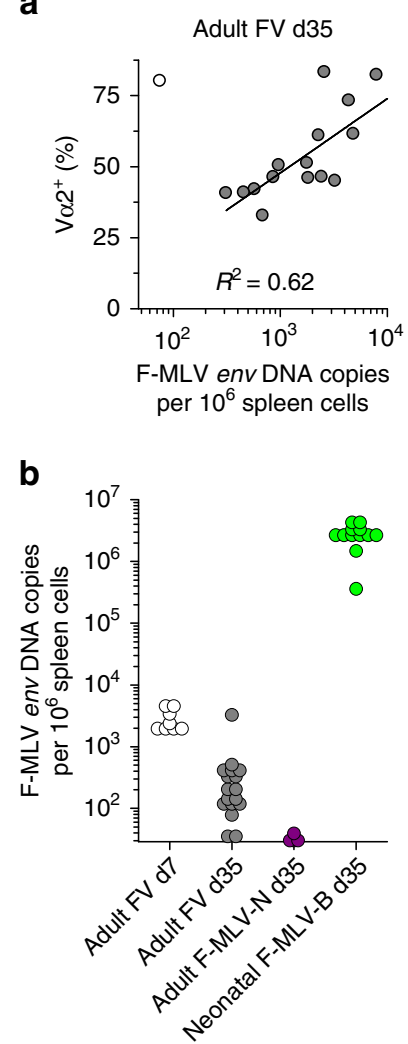

Figure 3 | Effect of infection kinetics on the clonal composition of virusspecific CD4 ${ }^{+} \mathbf{T}$ cells. (a) Frequency of $\mathrm{V} \alpha 2{ }^{+}$cells within env-reactive donor EF4. $1 \mathrm{CD} 4{ }^{+}$T cells plotted against copies of F-MLV env DNA in the spleens of recipient mice 35 days after adoptive T-cell transfer and FV infection. Symbols represent individual mice. The mouse represented with an open symbol was excluded from the regression analysis. (b) Copies of F-MLV env DNA in the spleens of recipient mice that were infected as adults with FV or F-MLV-N or as neonates with F-MLV-B. All recipients received EF4.1 $\mathrm{CD}^{+}{ }^{+} \mathrm{T}$ cells as adults and analysed at the indicated time point after T-cell transfer. Each symbol is an individual mouse. The dashed line denotes the detection limit. (c) Absolute numbers (top) and $V \alpha$ composition (bottom) of env-reactive donor EF4.1 CD4 ${ }^{+}$T cells in the spleens of recipient mice after T-cell adoptive transfer in adult mice either infected with F-MLV-N at the time of T-cell transfer or with F-MLV-B as neonates $(n=4-15$ mice per time point; $P<0.001$ between the two types of host on days 14-35, Mann-Whitney rank sum test). Closed symbols are the means ( \pm s.e.m.); open symbols are individual mice; the dashed line represents the frequency of $\mathrm{V} \alpha 2^{+}$cells in preimmune env-reactive EF4.1 CD4 ${ }^{+} \mathrm{T}$ cells. the product of the $F v 1^{\mathrm{b}}$ allele and quickly eliminated from these mice 28 (Fig. 3b). In addition, we infected neonatal B6 mice with B-tropic F-MLV (F-MLV-B), which results in life-long high-level infection (Fig. 3b) and used them 6-8 weeks later as T-cell hosts. The magnitude of the EF4.1 CD4 ${ }^{+}$T-cell response to adult F-MLV-N or neonatal F-MLV-B infection was proportional to the degree of viral replication in these two infections (Fig. 3c). However, the frequency of $\mathrm{V} \alpha 2$ clonotypes in virus-specific donor $\mathrm{CD}^{+} \mathrm{T}$ cells declined quickly and homogeneously in all recipients transiently infected with F-MLV-N, whereas it remained very high in all recipients with non-resolving F-MLV-B infection (Fig. 3c). These results demonstrated that the clonotypic evolution of the EF4.1 $\mathrm{CD}^{+}{ }^{+}$T-cell response to retroviral infection was directly determined by the kinetics of viral replication and that higher antigen levels promoted, rather than hindered, clonotypes with higher antigen sensitivity.

B cells mediate clonal replacement in $\mathrm{CD4}^{+} \mathrm{T}$ cells. $\mathrm{T}$ cells recognize viral antigens only through APCs. Thus, we reasoned that the effect of viral antigen availability on the composition virus-specific $\mathrm{CD}_{4}{ }^{+} \mathrm{T}$ cells involved APCs. In addition to acting as APCs to T cells, B cells mount their own antigen-specific and non-specific response to viral infection with distinct temporal kinetics. In response to $\mathrm{FV}$ infection, the frequency of germinal center $\mathrm{CD} 38^{\text {lo }} \mathrm{GL} 7^{\text {hi }}$ cells within $\mathrm{IgD}^{\mathrm{lo}} \mathrm{CD} 19^{+} \mathrm{B}$ cells slowly increased starting from day 10 post infection and continuing until the last time point studied (day 35; Fig. 4a,b). Interestingly, the rise in activated $\mathrm{B}$ cells temporally matched the decline in $\mathrm{V} \alpha 2$ clonotypes in virus-specific donor $\mathrm{CD} 4^{+} \mathrm{T}$ cells in $\mathrm{FV}$ infection (Fig. $1 \mathrm{~b}$ and Fig. 4a). To examine a possible link, we compared hosts infected with FV with those coinfected with FV and lactate dehydrogenase-elevating virus (LDV), which has been previously shown to induce rapid polyclonal B-cell activation and enhance FV replication ${ }^{29}$. As expected, FV-LDV coinfection greatly accelerated the rise in germinal center $\mathrm{B}$ cells and induced higher expression of MHC II on all B cells (Fig. 4a,c). Importantly, FV-LDV coinfection resulted in significantly faster decline in the frequency of $\mathrm{V} \alpha 2$ clonotypes in virus-specific EF4.1 $\mathrm{CD} 4{ }^{+} \mathrm{T}$ cells, than FV infection (Fig. 4d), supporting a role for polyclonally activated $\mathrm{B}$ cells.

As coinfection with LDV is likely to affect multiple processes during the response to $\mathrm{FV}$, we next examined if the accelerated $\mathrm{V} \alpha 2$ clonotype decline required T cell-B cell interaction. To this end, we used EF4.1 CD4 ${ }^{+} \mathrm{T}$ cells deficient in Sh2d1a (encoding SAP, multiple signalling lymphocyte activation moleculeassociated protein), which is required for prolonged $\mathrm{CD} 4{ }^{+}$ $\mathrm{T}$ cell-B cell contacts ${ }^{30}$. Although SAP deficiency in donor $\mathrm{CD} 4^{+} \mathrm{T}$ cells had little effect on their numerical response to FV infection or FV-LDV coinfection, it completely negated the accelerated decline of $\mathrm{V} \alpha 2$ clonotypes in virus-specific EF4.1 $\mathrm{CD} 4{ }^{+} \mathrm{T}$ cells by LDV (Fig. $4 \mathrm{~d}$ ).

As T-cell-specific SAP deficiency shortens, but does not abolish $\mathrm{T}$ cell-B cell interaction ${ }^{30}$, we next used mice deficient in B cells $\left(\mathrm{Ighm}^{-/-}\right)$as hosts. In the complete absence of B cells, highavidity $\mathrm{V} \alpha 2$ clonotypes were induced and maintained at high levels throughout FV infection or FV-LDV coinfection (Fig. 4e). To dissociate a possible effect of B cells as APCs from other B-cell-dependent processes in the host (for example, splenic structure) we reconstituted non-irradiated $\mathrm{Ighm}^{-1-}$ hosts with either MHC II-sufficient or -deficient B cells (Supplementary Fig. 3). Following FV-LDV coinfection, MHC II-sufficient, but not -deficient $B$ cells significantly reduced the frequency of highavidity V $\alpha 2$ clonotypes, in a dose-dependent manner, without affecting the total virus-specific donor $\mathrm{CD}^{+}$T-cell numbers (Fig. 4f). Therefore, clonotypic replacement of V 22 virus-specific 

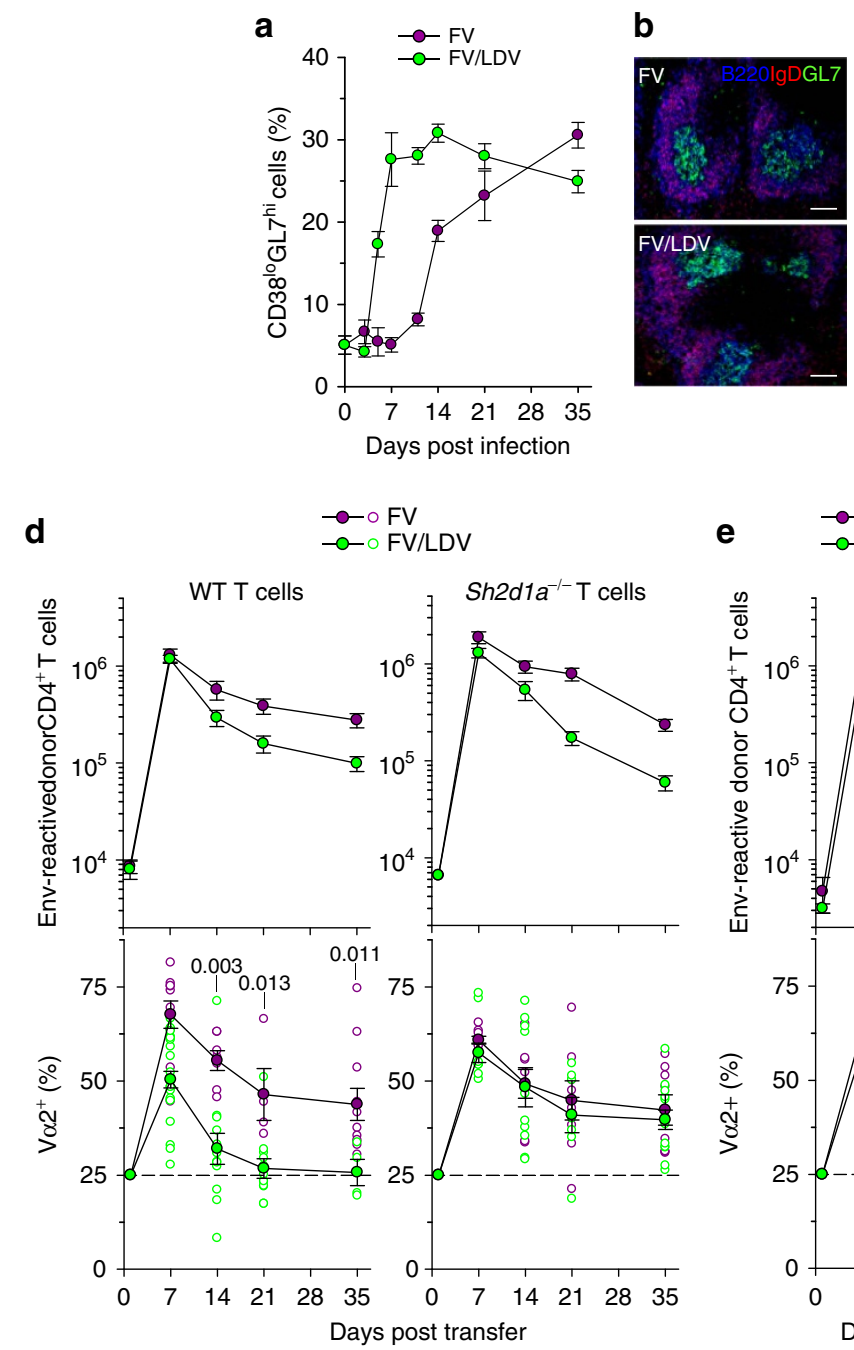

C $\square$ Uninfected $\square$ Infected
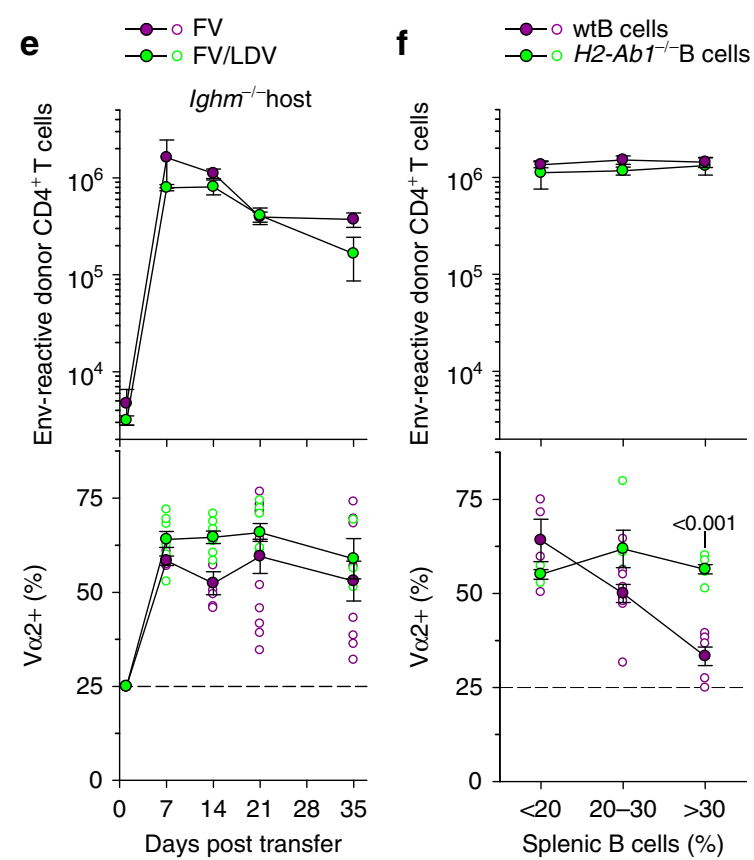

Figure 4 | Clonal replacement in virus-specific CD4 ${ }^{+} \mathbf{T}$ cells requires cognate B-cell interaction. (a) Frequency of germinal center-phenotype $\left(\mathrm{CD} 38^{\mathrm{lo}} \mathrm{GL} 7^{\text {hi }}\right)$ cells in $\mathrm{CD} 19{ }^{+} \mathrm{IgD}{ }^{\mathrm{lo}}$ splenic $\mathrm{B}$ cells over the course of $\mathrm{FV}$ infection or $\mathrm{FV} / \mathrm{LDV}$ coinfection $(n=4-13 ; P<0.002$ between the two infections on days 5-14, Mann-Whitney rank sum test). (b) Germinal center $\left(B 220^{+} \operatorname{lgD}-\mathrm{GL}^{+}\right.$) presence in the spleen of the same mice in a 35 days post infection. The scale bar is $250 \mu \mathrm{m}$. (c) MHC II expression in B220 ${ }^{+} \mathrm{CD} 19+$ B cells from the spleen of the same mice in a 7 days post infection, compared with uninfected mice. ( $P=0.0016$ between the two infections, Student's $t$-test). (d) Absolute numbers (top) and $V \alpha$ composition (bottom) of WT (left) or Sh2 $\mathrm{dla}^{-/-}$(right) env-reactive donor EF4.1 CD4 ${ }^{+}$T cells in the spleens of recipient mice after adoptive T-cell transfer and FV infection ( $n=3-8$ mice per time point) or FV/LDV coinfection ( $n=3-9$ mice per time point). (e) Absolute numbers (top) and $V \alpha$ composition (bottom) of env-reactive donor EF4.1 $\mathrm{CD}^{+}{ }^{+} \mathrm{T}$ cells in the spleens of WT or $1 \mathrm{ghm}{ }^{-/-}$recipient mice after adoptive T-cell transfer and FV infection ( $n=4-11$ mice per time point) or FV/LDV coinfection ( $n=4-23$ mice per time point). (f) Absolute numbers (top) and $\mathrm{V} \alpha$ composition (bottom) of env-reactive donor EF4.1 CD4 ${ }^{+} \mathrm{T}$ cells in the spleens of $I \mathrm{ghm}^{-/-}$mice previously reconstituted with WT $(n=18)$ or H2-Ab1 ${ }^{-/}-$B cells $(n=14) 14$ days after adoptive T-cell transfer FV/LDV coinfection, plotted against the level of B-cell reconstitution. In $\mathbf{d}-\mathbf{f}$, closed symbols are the means ( \pm s.e.m.); open symbols are individual mice.

$\mathrm{CD} 4{ }^{+} \mathrm{T}$ cells over the course of infection required cognate $\mathrm{T}$ cell-B cell interaction.

$\mathrm{CD}^{+}$T-cell clonal replacement affects all Th subsets. Follicular helper (Tfh) cells rely on B-cell interaction and the germinal center response for their differentiation and persistence. Thus, it was theoretically possible that B cells promoted Tfh differentiation of particular T-cell clonotypes selectively, whose representation was subsequently proportional to the magnitude of the germinal center response. To test the requirement for the physical germinal center structures, we used Tnfrsfla-deficient hosts, which show incomplete segregation of T-cell and B-cell areas in secondary lymphoid organs and are unable to mount a germinal center response (Fig. 5a,b). In comparison with those in
WT hosts, higher numbers of virus-specific donor CD4 ${ }^{+} \mathrm{T}$ cells were recovered from Tnfrsfla-deficient hosts throughout the response (Fig. 5c). Importantly, however, the $\mathrm{V} \alpha 2$ and $\mathrm{V} \alpha 3$ composition of donor CD4 ${ }^{+} \mathrm{T}$ cells was comparable between the two types of hosts (Fig. 5c), ruling out a requirement for germinal centres in the clonotypic evolution of virus-specific CD4 ${ }^{+} \mathrm{T}$ cells.

To directly examine if replacement of $\mathrm{V} \alpha 2$ clonotypes was owing to preferential differentiation into Tfh cells, we conditionally ablated $B c l 6$ in donor $\mathrm{CD}^{+} \mathrm{T}$ cells, precluding their Tfh differentiation. EF4.1 CD4 ${ }^{+} \mathrm{T}$ cells were isolated from mice carrying a Cre-conditional $B c l 6\left(B c l \sigma^{c}\right)$ and a YFP reporter (Gt(ROSA)26Sor ${ }^{\mathrm{YFP}}$ ) allele and also expressed Cre under the control of the Tnfrsf4 promoter (Tnfrsf4 ${ }^{\mathrm{Cre}}$ ), thus initiating YFP expression and Bcl6 deletion only upon T-cell activation ${ }^{31}$ (Fig. 5d,e). EF4.1 CD4 ${ }^{+} \mathrm{T}$ cells with either WT or Bcl6 ${ }^{\mathrm{C}}$ alleles, 


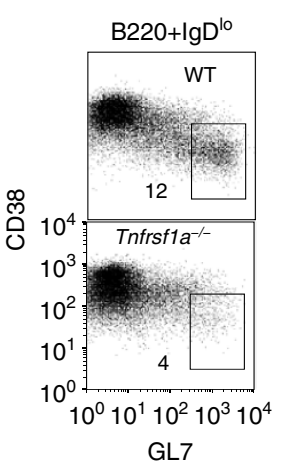

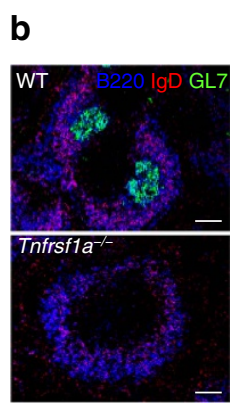

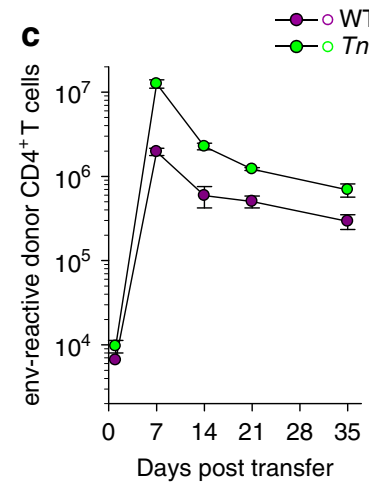

Days post transfer

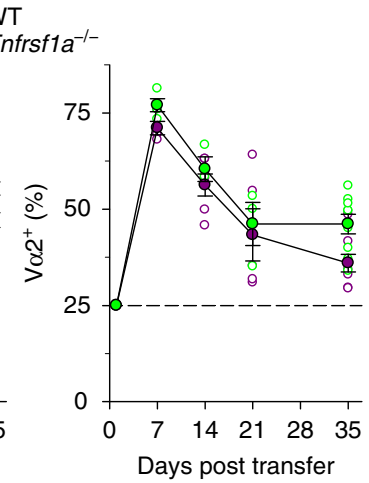

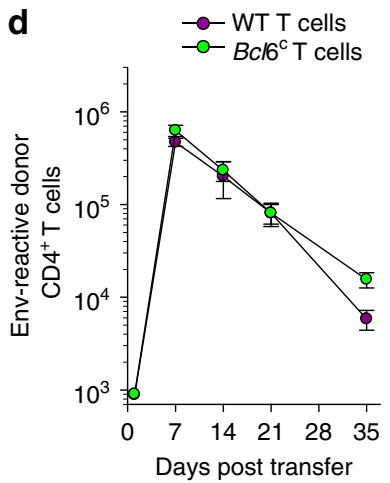
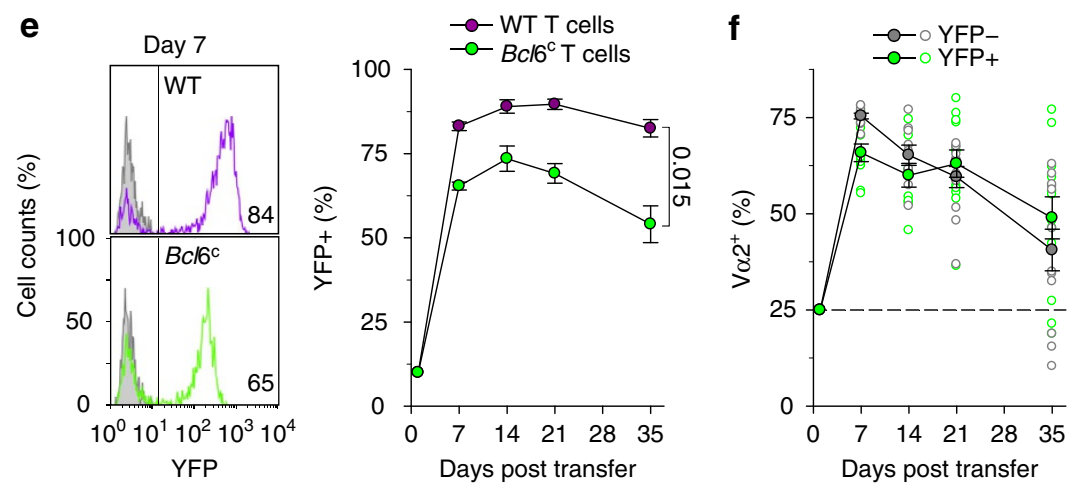

Figure 5 | Clonal replacement in virus-specific CD4 ${ }^{+} \mathbf{T}$ cells independently of Bcl6-dependent Tfh differentiation or germinal center reaction.

(a) Frequency of germinal center-phenotype $\left(\mathrm{CD} 38^{\mathrm{lo}} \mathrm{GL} 7^{\text {hi }}\right)$ cells in CD19+ ${ }^{+} \mathrm{gD}{ }^{\text {lo }}$ splenic B cells 35 days after FV infection of WT or Tnfrsf1a ${ }^{-/-}$mice $(n=3)$. (b) Germinal center $\left(B 220^{+} \mid g D^{-} G L 7^{+}\right.$) presence in the spleen of the same mice in a. The scale bar is $250 \mu \mathrm{m}$. (c) Absolute numbers (left) and $\mathrm{V} \alpha$ composition (right) of env-reactive donor EF4.1 CD4 ${ }^{+}$T cells in the spleens of WT or Tnfrsf1a ${ }^{-/}$- recipient mice after adoptive T-cell transfer and FV infection ( $n=3-9$ mice per time point). (d) Absolute numbers of WT or Bcl6-conditional ( $B c / 6^{c}$ ) env-reactive donor EF4.1 CD4 ${ }^{+}$T cells, additionally carrying the Tnfrsf4 ${ }^{\mathrm{Cre}}$ and Gt(ROSA)26Sor YFP alleles, in the spleens of recipient mice after adoptive T-cell transfer and FV infection ( $n=3-9$ mice per time point). (e) YFP expression on day 7 (left) and frequency of YFP ${ }^{+}$cells over time (right) in the same env-reactive donor EF4.1 CD4 ${ }^{+} \mathrm{T}$ cells as in d. (f) $V \alpha 2$ frequency in $\mathrm{YFP}^{+}$and YFP ${ }^{-}$Bcl6-conditional env-reactive donor EF4.1 CD4 ${ }^{+}$T cells as in $\mathbf{d}$. In c-f, closed symbols are the means ( \pm s.e.m.); open symbols are individual mice.

exhibited typical response kinetics upon transfer into FV-infected hosts (Fig. 5d). However, the frequency of $\mathrm{YFP}^{+}$cells in virus-specific donor $\mathrm{CD} 4^{+} \mathrm{T}$ cells was significantly lower in EF4.1 CD4 ${ }^{+} \mathrm{T}$ cells with the $B c l 6^{\mathrm{c}}$ than the WT allele (Fig. 5e), indicating that $\mathrm{Bcl} 6$ is required for the persistence of at least some virus-specific $\mathrm{CD}^{+} \mathrm{T}$ cells. Nevertheless, the clonotypic composition of $\mathrm{YFP}^{+}$(Bcl6-deleted) virus-specific donor $\mathrm{CD}^{+} \mathrm{T}$ cells was comparable with that of the $\mathrm{YFP}^{-}$fraction (Fig. 5f). Thus, preventing Bcl6-dependent Tfh differentiation did not prevent the switch from $\mathrm{V} \alpha 2$ to $\mathrm{V} \alpha 3$ clonotypes $(P=0.016$ $\mathrm{V} \alpha 2$ frequency in $\mathrm{YFP}^{+}$cells between days 7 and 35 , Mann-Whitney rank sum test).

These findings argued against the possibility that clonotypic replacement of $\mathrm{V} \alpha 2$ clonotypes with Traj31-using V $\alpha 3$ clonotypes over the course of $\mathrm{FV}$ infection was owing to selective differentiation into the Tfh subset, whose long-term stability may differ from that of other Th subsets. This led us to consider an alternative hypothesis: instead of all virus-specific clonotypes engaging synchronously and Traj31-using V $\alpha 3$ clonotypes surviving longer than other clonotypes, it was possible that Traj31-using V $\alpha 3$ clonotypes were simply recruited with delayed kinetics.

Asynchronous expansion of high- and low-avidity clonotypes. The extensive variability in the frequency of $V \alpha 3$ clonotypes between separate hosts during FV infection (Fig. 1b), and the 'private' nature of distinct Traj31-using V $\alpha 3$ clonotypes expanding in different hosts (Fig. 1c) hindered kinetic analyses of the deep-sequencing data. To establish conclusively the kinetics of recruitment and expansion of a Traj31-using V $\alpha 3$ clonotype in response to FV infection, we transferred cohorts of allotypically marked monoclonal EV $23 \mathrm{~T}$ cells into WT hosts that were infected with FV or coinfected with FV and LDV, either 10 days previously or on the day of T-cell transfer (Fig. 6a,b). EV $\alpha 3$ $\mathrm{T}$ cells transferred at the time of $\mathrm{FV}$ infection or FV/LDV coinfection expanded minimally 7 days later and became undetectable by day 21 post transfer (Fig. 6a,b). Also, EV $\alpha 3 \mathrm{~T}$ cells transferred on day 10 of FV infection expanded modestly 7 days after transfer and contracted to low numbers thereafter (Fig. 6a,b). This pattern contrasted with strong expansion ( $>100$-fold) of EV $33 \mathrm{~T}$ cells 7 days after transfer into hosts coinfected with FV and LDV 10 days previously (Fig. 6a,b), when B-cell activation had peaked (Fig. 4a). For comparison, EF4.1T cells were also transferred into hosts that were coinfected with FV and LDV, either 10 days previously or on the day of T-cell transfer, and maximal expansion of both $\mathrm{V} \alpha 2$ and $\mathrm{V} \alpha 3$ clonotypes was already observed the first 7 days of infection (Fig. 6c). These results demonstrated that EV $\alpha 3 \mathrm{~T}$ cells were recruited later than higher-avidity virus-specific clonotypes and only in conditions where B cells were activated.

Later recruitment, as opposed to longer survival, of EV $\alpha 3 \mathrm{~T}$ cells further argued that B-cell presentation did not necessarily instill qualitative differences in $\mathrm{CD} 4^{+}{ }^{+}$-cell clonotypes, but rather expanded the number of available APCs to the point where clonotypic competition was alleviated and lower-avidity T-cell clonotypes could be expanded. Indeed, recruitment and expansion of lower-avidity T-cell clonotypes depended on the 
a

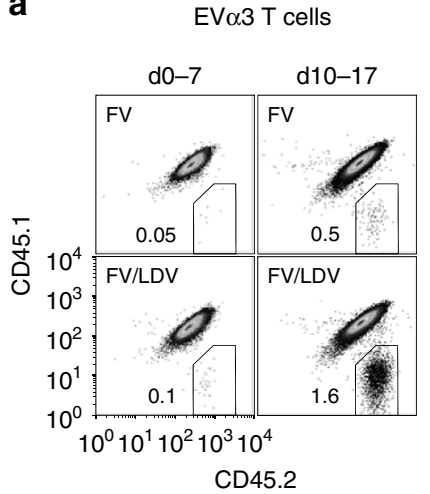

b

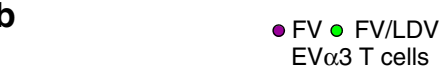

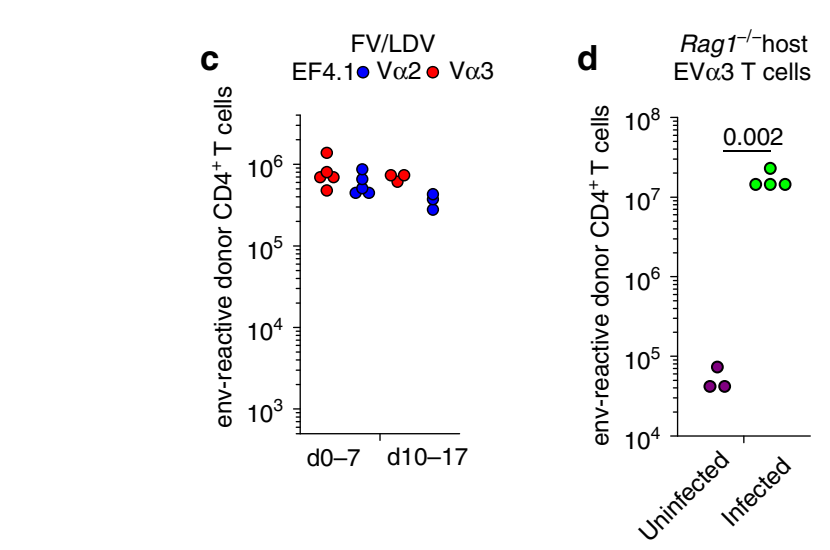

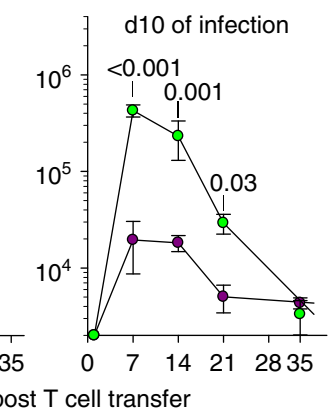

Figure 6 | Asynchronous expansion of higher- and lower-avidity clonotypes. (a) Flow cytometric detection of CD45.2 ${ }^{+}$donor EV $\alpha 3$ Rag $1^{-/-} E m v 2^{-/-}$ CD4 ${ }^{+}$T cells and CD45.1 ${ }^{+}$CD45.2 ${ }^{+}$host CD4 ${ }^{+}$T cells following transfer either on the day of infection (d0 of infection, d0-7) or 10 days after infection (d10 of infection, d10-17) with FV or FV/LDV. In both setups, T-cell expansion was analysed 7 days after transfer $(n=5-9)$. (b) Absolute number of env-reactive donor EV $\alpha 3 \mathrm{CD} 4^{+}$T cells recovered from the spleens of the same recipients described in a over the course of FV infection or FV/LDV coinfection (nd, not detected). (c) Absolute number of $\mathrm{V} \alpha 2$ or $\mathrm{V} \alpha 3$ env-reactive donor EF4.1 CD4 ${ }^{+} \mathrm{T}$ cells recovered from the spleens of FV/LDV infected recipients 7 days after transfer either on the day of infection (d0-7) or 10 days after infection (d10-17). (d) Numbers of EV $\alpha 3$ T cells recovered 7 days after transfer from the spleens of lymphocyte-deficient Rag1-/- Emv2 ${ }^{-/-}$recipients that were either left uninfected or were F-MLV-B-infected. Each symbol represents an individual mouse.

availability of activated B cells only in the presence of higheravidity T-cell competitors. In contrast to their lack of expansion in FV-infected WT host (Fig. 6a,b), EV $\alpha 3 \mathrm{~T}$ cells expanded markedly when transferred into FV-infected Rag1-1- hosts (Fig. 6d). These results were consistent with a quantitative, rather than qualitative effect of B-cell presentation on the expansion of lower-avidity $\mathrm{CD}_{4}{ }^{+} \mathrm{T}$-cell clonotypes, whereby the B-cell response gradually increases APC availability, which progressively recruits lower-avidity $\mathrm{T}$-cell clonotypes, thus diversifying the $\mathrm{CD} 4{ }^{+} \mathrm{T}$-cell response.

B cells promote TCR diversity in various T-cell responses. Our model would also predict that following infections or immunizations where B cells are dominant APCs, lower-avidity clonotypes would be induced, in addition to higher-avidity clonotypes. To test this prediction, we compared the dependence on B cells of EF4.1 CD4 ${ }^{+}$T-cell priming by the replicationattenuated F-MLV-N vaccine virus and fully competent FV, which induce lower- and higher-avidity responses, respectively (Fig. $1 \mathrm{~b}$ and Fig. 3c). Indeed, CD4 ${ }^{+}$T-cell priming by F-MLV-N, but not FV, was significantly compromised in the absence of $\mathrm{B}$ cells (Fig. 7a). Similarly, priming with a replication-defective human Adenovirus 5 (Ad5)-based vector, expressing F-MLV gp70 (Ad5.pIX-gp70), which has previously found to induce primarily lower-avidity responses ${ }^{16}$, was also compromised in the absence of B cells (Fig. 7a). Therefore, vaccines that rely on B-cell presentation induce lower-avidity $\mathrm{CD} 4{ }^{+} \mathrm{T}$-cell responses.

The applicability of this model was also tested in two additional immunization regimens. WT or $\mathrm{Ighm}^{-1}$ hosts were challenged with FBL-3 cells, a FV-induced tumour cell line that expresses F-MLV gp70, but does not produce infectious viral particles ${ }^{32}$. Adoptively transferred EF4.1 $\mathrm{CD}^{+} \mathrm{T}$ cells responded with a high frequency of high-avidity $\mathrm{V} \alpha 2$ virus-specific $\mathrm{CD} 4{ }^{+} \mathrm{T}$-cell clonotypes in both types of host at peak (Fig. 7b). Importantly, this frequency remained significantly higher in $\mathrm{Ighm}^{-l}$ than in WT hosts as the response progressed (Fig. 7b). Moreover, the clonotypic replacement of $\mathrm{V} \alpha 2$ virus-specific $\mathrm{CD} 4{ }^{+} \mathrm{T}$ cells was observed following env $\mathrm{v}_{124-138}$ peptide immunization of WT, but not $I g \mathrm{hm}^{-1}$ - hosts, highlighting the requirement for B cells in the process. Thus, following either tumour challenge or peptide immunization, B cells were required for the full clonotypic diversity of the $\mathrm{CD} 4^{+} \mathrm{T}$-cell response.

B cells balance TCR diversity during T-cell reconstitution. Our model implied that B-cell presentation should promote TCR diversity not only in response to infection or immunization, but also in any setting where $\mathrm{CD} 4{ }^{+} \mathrm{T}$-cell clonotypic competition is likely to operate based on TCR avidity. To examine if this were the case, we monitored TCR repertoire skewing during $\mathrm{CD} 4^{+}$ T-cell reconstitution of lymphopenic hosts, which selects distinct clonotypes according to TCR avidity. Hosts lacking only T cells 

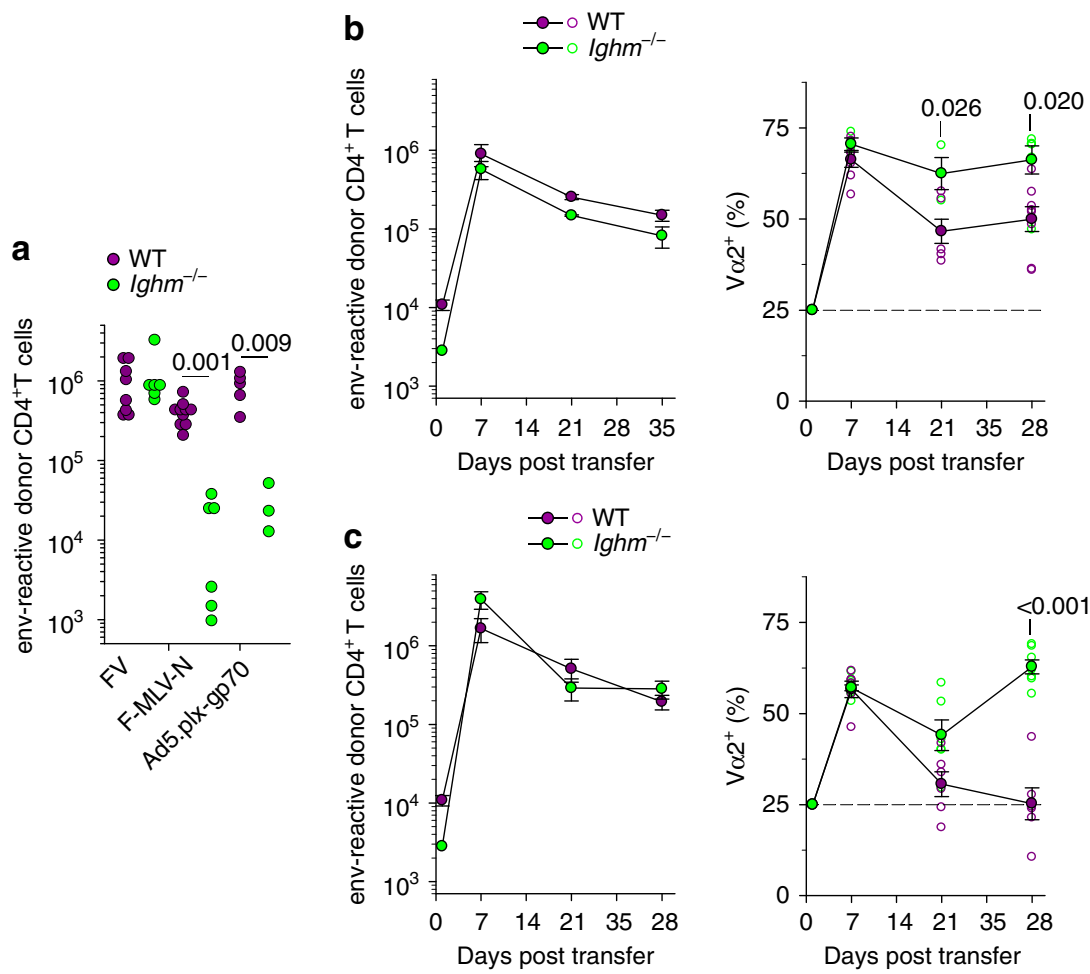

Figure 7 | B-cell-dependent expansion of lower-avidity CD4 ${ }^{+}$T-cell clonotypes in diverse infection or immunization settings. (a) Absolute number of env-reactive donor EF4.1 CD4 ${ }^{+}$T cells 7 days after transfer into WT or Ighm ${ }^{-1}$ - recipient mice infected either with FV or F-MLV-N or immunized with Ad5.pIX-gp70. Each symbol is an individual mouse. (b) Absolute numbers (left) and $V \alpha$ composition (right) of env-reactive donor EF4.1 CD4 ${ }^{+}$T cells in the spleens of WT or $1 \mathrm{ghm}^{-1-}$ recipient mice after adoptive T-cell transfer and FBL-3 tumor challenge $(n=3-8$ mice per time point). (c) Absolute numbers (left) and $\mathrm{V} \alpha$ composition (right) of env-reactive donor EF4.1 CD4 ${ }^{+}$T cells in the spleens of WT or lghm ${ }^{-/-}$recipient mice after adoptive T-cell transfer and env $_{122-141}$ peptide immunization ( $n=6-9$ mice per time point).

a

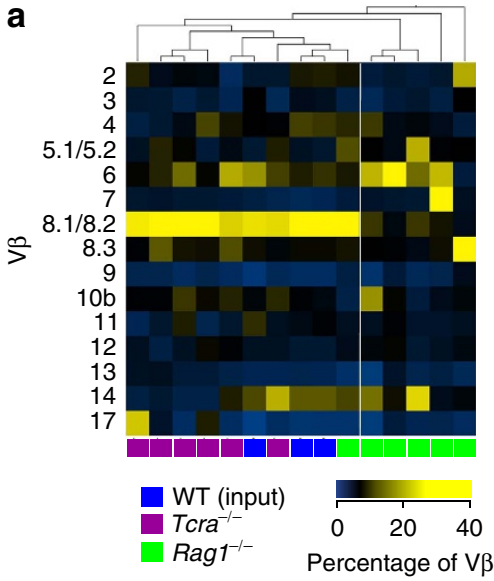

b

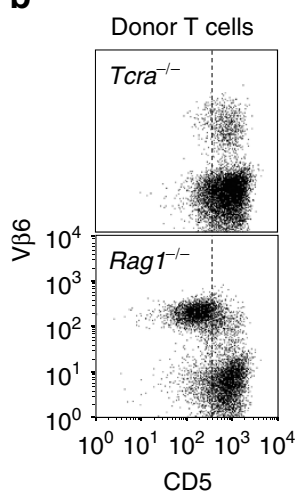

C

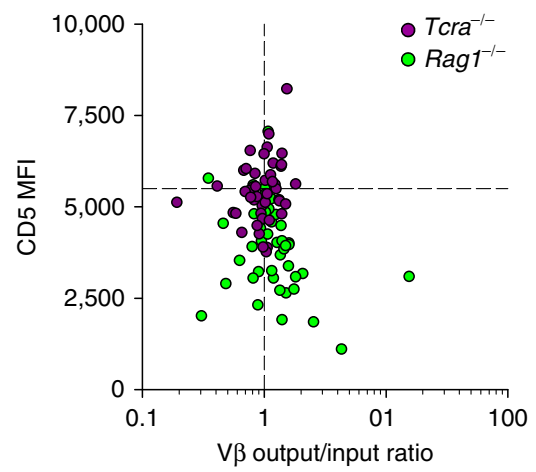

d

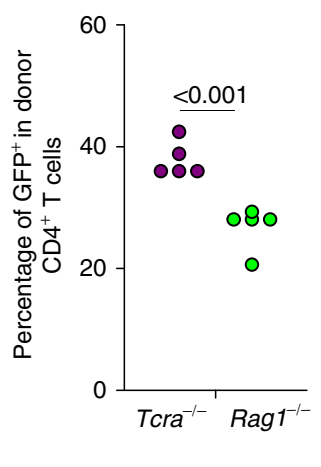

Figure 8 | Balanced TCR diversity during CD4 ${ }^{+}$T-cell reconstitution requires B cells. (a) Heat-map of hierarchically-clustered WT donors and Tcra ${ }^{-/-}$ or Rag $1^{-/}-E m v 2^{-/}-$recipients of purified CD4 ${ }^{+}$T cells according to the frequency of the indicated V $\beta$ family before (WT (input)) or 21 days after T-cell reconstitution ( $\mathrm{Tcra}^{-/-}$or Rag1-/-). Each column is an individual mouse. (b) Flow cytometric detection of CD5 and V $\beta 6$ expression in donor CD4 ${ }^{+} \mathrm{T}$ cells from the same recipients as in $\mathbf{a}$. (c) CD5 median fluorescent intensity (MFI) plotted against the divergence from the input frequency of each detected $\mathrm{V} \beta$ family in donor $\mathrm{CD}^{+}{ }^{+} \mathrm{T}$ cells from the same recipients as in $\mathbf{a}$. Only three recipients are plotted for clarity. The horizontal dashed line represents the CD5 MFI in control cells ( $P<0.001$ between CD5 levels in T cells from the two types of host, Mann-Whitney rank sum test). (d) Frequency of GFP + cells

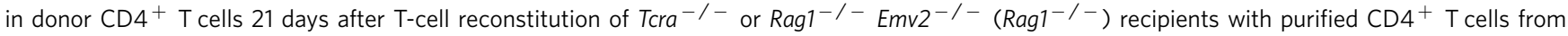
Nur77-GFP transgenic donors. Each symbol represents an individual mouse.

$\left(\right.$ Tcra $\left.^{-I^{-}}\right)$or both $\mathrm{T}$ and $\mathrm{B}$ cells $\left(\operatorname{Rag} 1^{-I^{-}}\right)$were reconstituted by adoptive transfer of fully polyclonal purified $\mathrm{CD} 4{ }^{+} \mathrm{T}$ cells and usage of different families of $\mathrm{V} \beta$ chains was compared between the input and expanded population in the respective host 21 days later (Fig. 8a). T-cell reconstitution of $\mathrm{Tcra}^{-/-}$hosts preserved the pattern of $\mathrm{V} \beta$ usage in the input population, as the two were indistinguishable by unsupervised clustering (Fig. 8a). In contrast, T-cell reconstitution of $\operatorname{Rag} 1^{-1}$ - hosts resulted in considerable skewing in $\mathrm{V} \beta$ usage in 5 out of 6 such hosts, which clustered separately from all other hosts (Fig. 8a). TCR V $\beta$ usage skewing 
was not related to differences in overall $\mathrm{T}$-cell reconstitution between $\mathrm{Tcra}^{-1-}$ and Rag1-1- hosts, as it also characterized hosts with comparable numbers of expanded $\mathrm{CD} 4{ }^{+} \mathrm{T}$ cells (Supplementary Fig. 4). TCR repertoire skewing in $R a g 1^{-1-}$ hosts was owing to 'private' expansion of distinct $\mathrm{V} \beta$ families in individual mice (Fig. 8a), suggestive of TCR driven responses. Moreover, levels of CD5 expression were significantly reduced in $\mathrm{CD}^{+}{ }^{+} \mathrm{T}$ cells expanded in Rag1 ${ }^{-/}-$hosts in comparison with those expanded in Tcra ${ }^{-1-}$ hosts (Fig. 8b,c), indicating insufficient MHC II-derived signals in Rag1 ${ }^{-1}-$ hosts $^{33,34}$. Indeed, use of the Nur77-GFP reporter for TCR signalling ${ }^{35}$ revealed that $\mathrm{CD}^{+}{ }^{+} \mathrm{T}$ cells received stronger overall TCR signals in Tcra ${ }^{-1-}$ than Rag $^{-1}-$ hosts (Fig. 8d), suggesting that B cells were necessary to provide CD4 ${ }^{+} \mathrm{T}$ cells with optimal TCR signal, which would be critical for the expansion of lower-avidity clonotypes. Thus, balanced representation of TCR clonotypes, at least at the level of distinct $\mathrm{V} \beta$ families during $\mathrm{T}$-cell reconstitution, required $\mathrm{B}$ cells.

\section{Discussion}

Our findings highlight the prominent role for the TCR in setting the clonotypic hierarchy during the $\mathrm{CD}^{+}$T-cell response, suggested by studies in many systems ${ }^{36}$. They lend support to the recent recognition of the importance of self-reactivity, rather than affinity for antigen, in determining the fate of $\mathrm{CD} 4^{+}$and $\mathrm{CD} 8^{+}$ $\mathrm{T}$ cells during the response to foreign antigens ${ }^{11,12,37}$. However, they further uncover the role of B-cell presentation as a powerful T-cell-extrinsic factor that can reverse TCR sensitivity-based hierarchies. Indeed, although $\mathrm{T}$-cell clonal selection driven by TCR affinity for self or foreign antigens progressively narrows the responding TCR repertoire, B cells are critical in preserving diversity of the antigen-selected TCR repertoire.

The progression of the $\mathrm{CD} 4{ }^{+} \mathrm{T}$-cell response is generally expected to enrich for high-avidity clonotypes, accentuated by diminishing antigen levels, a notion that is supported by experimental data ${ }^{10,38-40}$. The central importance of TCR signal strength in memory formation would gradually reduce the diversity to a few or theoretically the one clonotype with the highest avidity. However, enrichment for high-avidity clonotypes is not consistent with either continuous clonal replacement during chronic $\mathrm{T}$-cell responses ${ }^{41}$ or following secondary responses ${ }^{10,42,43}$. Moreover, memory $\mathrm{CD} 4{ }^{+} \mathrm{T}$-cell populations with lower overall avidity than the primary response have also been found in response to Salmonella typhimurium infection ${ }^{44}$, and a mouse adenocarcinoma-associated antigen ${ }^{45}$.

The contribution of low-avidity $\mathrm{CD} 4{ }^{+} \mathrm{T}$ cells to the antigenspecific response may be underestimated owing to technical difficulties in detecting such clonotypes by pMHC II tetramers ${ }^{23,24}$. Nevertheless, an equally plausible explanation for variable participation of lower-avidity $\mathrm{CD} 4{ }^{+} \mathrm{T}$-cell clonotypes is likely related to the nature of antigen and the range of TCR affinities it elicits. T-cell-extrinsic effects on TCR clonotypic composition will be particularly pronounced when TCR affinity for antigen might not be strong enough to overcome these effects. For example, self-tolerance is likely to reduce the overall avidity of T-cell responses to tumour-associated or self-antigens or to pathogen antigens with significant similarity with self, such as retroviruses ${ }^{1,2}$.

Another important variable in studies of TCR repertoire changes over the course of the $\mathrm{CD} 4{ }^{+} \mathrm{T}$-cell response is the definition of the exact property of antigen-selected TCRs. Although there is general consensus that $\mathrm{CD} 4^{+} \mathrm{T}$ cells are selected on the basis of TCR signal strength in response to antigen, the underlying factors responsible for differences in TCR sensitivity are still a matter of debate. TCR binding kinetics to antigenic pMHC II complexes have traditionally been considered as the dominant factor ${ }^{9,46}$. However, the importance of TCR self-reactivity in setting TCR responsiveness to antigen is increasingly appreciated $11,12,37$. Strong self-reactivity has been suggested to select for TCRs with improved binding kinetics to antigenic $\mathrm{pMHC}^{12}$ or to maintain a more efficient state of TCR signalling capacity, irrespective of affinity to antigenic $\mathrm{pMHC}^{11}$ Our results with the EV $\alpha 2$ and EV $\alpha 3$ TCRs are consistent with the latter notion, whereby differences in functional avidity and biological response correlate with self-reactivity rather than affinity to antigen ${ }^{11}$. However, as a direct mechanistic link between self-reactivity and antigen sensitivity was not examined in the current study, our results do not prove that differences in antigen sensitivity are indeed caused by differences in selfreactivity.

Although naive $\mathrm{CD} 4{ }^{+}$T-cell priming is thought to be initiated by DCs in the T-cell zone of secondary lymphoid organs, the contribution to maximal T-cell expansion of additional APCs and $B$ cells in particular, is well-recognized ${ }^{47}$. Our findings uncover an additional dimension to the antigen-presenting contribution of $\mathrm{B}$ cells, namely the dependence on the B-cell response itself. Indeed, B-cell-mediated expansion of lower-avidity $\mathrm{CD}^{+}$T-cell clonotypes was proportional to the degree of B-cell activation. Polyclonal B-cell activation is a feature of many viral infections or autoimmune diseases ${ }^{48,49}$. Notably, progression of human immunodeficiency virus- 1 infection is characterized by stepwise B-cell hyperactivation ${ }^{50}$, asynchronous expansion of $\mathrm{CD}^{+} \mathrm{T}$ cells specific to distinct epitopes ${ }^{51}$ and increase in virus-specific $\mathrm{CD} 4{ }^{+} \mathrm{T}$ cells with low functional avidity ${ }^{52}$. The observation that germinal center formation was redundant in this process and that expansion of lower-avidity $\mathrm{CD} 4{ }^{+} \mathrm{T}$-cell clonotypes correlated simply with the overall number of activated $\mathrm{B}$ cells in bone marrow chimeras, argue against a requirement for a specific B-cell subset. Moreover, efficient priming of lower-avidity CD4 ${ }^{+}$ T-cell clonotypes in the absence of $\mathrm{B}$ cells, when higher-avidity T-cell competitors were also absent, further argues against a unique type of antigen presentation by $B$ cells. Instead, these findings suggest that B cells, which outnumber DCs at steadystate by two orders of magnitude ${ }^{53}$ and which further expand in response to polyclonal and antigen-specific stimuli during infection, provide the necessary abundance of antigenic stimulation to allow priming and expansion of all clonotypes irrespective of TCR avidity.

Our findings also indicate an essential role for B cells in maintenance of a diverse and balanced TCR repertoire during $\mathrm{CD} 4{ }^{+}$T-cell reconstitution of lymphopenic hosts, typically driven by relatively low-avidity interactions with self- and environmental-antigens and selecting for clonotypes that receive the strongest TCR signal ${ }^{4}$. These results, thus, further support the idea that $B$ cells facilitate the expansion of lower-avidity clonotypes in general. Similarly, B cells may be critically required to initiate $\mathrm{CD} 4{ }^{+} \mathrm{T}$-cell responses to an autoantigen, which would be typically low-avidity. Interestingly, non-obese diabetic mice do not develop autoimmunity if they are rendered deficient in $\mathrm{B}$ cells ${ }^{54}$, and $\mathrm{B}$-cell reconstitution is required for the formation of a diverse TCR repertoire of $\mathrm{CD} 4{ }^{+} \mathrm{T}$-cell infiltrating the pancreas ${ }^{55}$. Although the overall avidity for pancreatic autoantigens of the different TCR repertoires was not examined $^{55}$, these studies offer further support to the notion that $\mathrm{B}$ cells increase the diversity of responding $\mathrm{CD} 4^{+}$ T cells.

$\mathrm{B}$ cells have long been suspected as drivers of the diversification of the $\mathrm{CD}^{+} \mathrm{T}$-cell response $\mathrm{s}^{56}$ and this study provides clear evidence of their involvement. In contrast to the intrinsic TCR affinity of distinct clonotypes and the clonotypic diversity in an individual, manipulating the degree of B-cell activation or antigen 
presentation to $\mathrm{CD} 4{ }^{+} \mathrm{T}$ cells, might provide a more amenable way of controlling the antigen-specific TCR repertoire during infection, vaccination or autoimmunity.

\section{Methods}

Mice. Inbred B6 and CD45.1 ${ }^{+}$congenic B6 (B6.SJL-Ptprc ${ }^{a} P e p 3^{b} /$ BoyJ) mice were originally obtained from The Jackson Laboratory (Bar Harbor, ME, USA). TCR $\beta$-transgenic EF4.1 mice ${ }^{21}$, Rag1-deficient $\left(\right.$ Ragl $\left.^{-1-}\right)$ mice $^{57}$, B-cell-deficient $\left(\mathrm{Ighm}^{-1-}\right)$ mice $^{58}$, SAP-deficient (Sh2d1a $\left.{ }^{-1-}\right)$ mice $^{59}$, TCR $\alpha$-deficient $\left(\mathrm{Tcra}^{-/-}\right)$mice $^{60}$, MHC II-deficient $\left(\mathrm{H2}-\mathrm{Abl}^{-/-}\right) \mathrm{mice}^{61}$, TNF receptor I-deficient (Tnfrsflia ${ }^{-1-}$ ) mice ${ }^{62}$, mice with an activatable YFP gene targeted into Gt(ROSA)26Sor (R26) locus ${ }^{63}$, mice with a targeted insertion of Cre recombinase into the Tnfrsf4 locus ${ }^{64}\left(\right.$ Tnfrsf $\left.^{C r e}\right)$, mice with a conditional Bcl6 allele ${ }^{65}\left(B^{6}{ }^{\mathcal{C}}\right)$, endogenous ecotropic MLV-deficient $\left(E m v 2^{-1-}\right)$ mice $^{23}$ and Nur77-GFP transgenic mice ${ }^{35}$ were all on the B6 genetic background. TCR $\alpha \beta$-transgenic EV $\alpha 2$ and EV $\alpha 3$ mice were created by conventional transgenesis (Supplementary Fig. 5). Briefly, cDNAs encoding the TCR $\alpha$ and TCR $\beta$ chains of the H5 and H18 envspecific $\mathrm{CD} 4{ }^{+} \mathrm{T}$-cell clones, respectively, from the polyclonal repertoire of EF4.1 mice $^{23}$, were cloned and inserted into the hCD2-VA expression cassette ${ }^{21}$. Each of these constructs was mixed with a construct encoding the TCR $\beta$ chain of EF4.1 mice and integrated into the DNA of fertilized B6 oocytes following pronuclear microinjection. Transgenic founders were identified by flow cytometry and genotyping for the presence of the TCR $\alpha$ and TCR $\beta$ transgenes. These new transgenic mice were crossed to $R a g 1^{-1-}$ mice, to preclude rearrangement and subsequent expression of productive endogenous TCR genes. They were additionally rendered deficient in Emv2, a single germ-line integration of a MLV found in B6 mice, and free from any Emv2-derived infectious MLVs that spontaneously arise in immunodeficient mice and have the potential to affect MLV-specific T-cell development and subsequent response ${ }^{66}$. Male or female mice were used in separate experiments and were gender-matched within experiments. Mice were used at 8-12 weeks of age, with the exception of neonatal infection, which was carried out on 1-2 day-old mice. All animal experiments were approved by the ethical committee of the Francis Crick institute, and conducted according to local guidelines and UK Home Office regulations under the Animals Scientific Procedures Act 1986 (ASPA)

Retroviral infection and immunization. The FV used in this study was a retroviral complex of a replication-competent B-tropic F-MLV and a replicationdefective SFFV. Stocks were propagated in vivo and prepared from the spleen of infected mice. A pool of $20 \mathrm{LDV}$-free BALB/c mice was infected with $\mathrm{FV}$, spleens were isolated 12 days later and homogenized $(10 \% \mathrm{w} / \mathrm{v})$ in phosphate-buffered saline. Aliquots were frozen and were subsequently used for infection. Mice received an inoculum of $\sim 1,000$ spleen focus-forming units of $\mathrm{FV}$ by intravenous injection. Stocks of F-MLV-B and F-MLV-N helper viruses were grown in $M u s$ dunni fibroblast cells. Mice received an inoculum of $\sim 10^{4}$ infectious units of F-MLV by intravenous injection. Neonatal F-MLV infection was performed by administering an inoculum of $\sim 4,000$ infectious units of F-MLV-B to 1-day-old mice by intraperitoneal injection. All stocks were free of Sendai virus, Murine hepatitis virus, Parvoviruses 1 and 2, Reovirus 3, Theiler's murine encephalomyelitis virus, Murine rotavirus, Ectromelia virus, Murine cytomegalovirus, $\mathrm{K}$ virus, Polyomavirus, Hantaan virus, Murine norovirus, Lymphocytic choriomeningitis virus, Murine adenoviruses FL and K87, Mycoplasma sp. and LDV. For coinfection of FV and LDV, a similarly prepared stock of FV additionally containing LDV was also used ${ }^{8}$. For peptide immunization, mice received an intraperitoneal injection of a total of $12.5 \mathrm{nmol}$ of synthetic env $\mathrm{en}_{124-138}$ peptide mixed in Sigma Adjuvant System. FBL-3 tumour challenge was carried out by intravenous injection of $3 \times 10^{6} \mathrm{FBL}-3$ cells. Ad5.pIX-gp70 stocks were prepared at a titre of $9 \times 10^{9}$ viral genomes per ml by infection of $293 \mathrm{~A}$ cells ${ }^{16}$.

Approximately $5 \times 10^{8}$ Ad5.pIX-gp70 viral genomes per mouse were administered intravenously.

F-MLV copy number analysis. DNA copy numbers of F-MLV were determined by real-time quantitative PCR (qPCR) on DNA samples isolated from the spleen cell suspensions from infected mice, using primers specific to F-MLV env DNA (125 bp product): forward $5^{\prime}$-AAGTCTCCCCCCGCCTCTA- $3^{\prime}$ and reverse

$5^{\prime}$-AGTGCCTGGTAAGCTCCCTGT- ${ }^{\prime}$. Signals were normalized for the amount of DNA used in the reactions based on amplification of the single-copy Ifnarl gene (150 bp product) with primers: forward $5^{\prime}$-AAGATGTGCTGTTCCCTTCC TCTGCTCTGA- $3^{\prime}$ and reverse $5^{\prime}$-ATTATTAAAAGAAAAGACGAGGCGA AGTGG-3'. Copy numbers were calculated with a $\Delta \Delta \mathrm{C}_{\mathrm{T}}$ method and are expressed as copies per million cells.

T-cell purification and adoptive transfer. Single-cell suspensions were prepared from the spleens and lymph nodes of donor CD45.1 $1^{+}$or CD $45.2^{+}$mice and $\mathrm{CD}^{+} \mathrm{T}$ cells were enriched using immunomagnetic positive selection (StemCell Technologies), at $>96 \%$ purity. A total of $1 \times 10^{6} \mathrm{CD} 4^{+} \mathrm{T}$ cells were injected into $\mathrm{CD} 45.1^{+} \mathrm{CD} 45.2^{+}$recipients via the tail vein. Where indicated, enriched EF4.1
$\mathrm{CD} 4{ }^{+} \mathrm{T}$ cells were further purified ( $>98 \%$ purity) by cell sorting, performed on MoFlo cell sorters (Dako-Cytomation, Fort Collins, CO, USA).

Bone marrow chimeras. Bone marrow cell suspensions were prepared by flushing the bone cavities of femurs and tibiae from donor mice with air-buffered Iscove's Modified Dulbecco's Media. MHC II-sufficient or -deficient bone marrow cells were injected separately into non-irradiated $I g \mathrm{gm}^{-1-}$ recipients. In this setting, only the missing lymphocyte population (B cells) is reconstituted ${ }^{31}$, creating B-cell-specific loss of MHC II. Each recipient received between $1 \times 10^{7}$ and $3 \times 10^{7}$ bone marrow cells. Mice were bled for assessment of reconstitution and were used for infection 8-10 weeks post bone marrow transfer.

Flow cytometry. Single-cell suspensions were stained with directly conjugated antibodies to surface markers (Supplementary Table 1), obtained from eBiosciences (San Diego, CA, USA), CALTAG/Invitrogen, BD Biosciences (San Jose, CA, USA) or BioLegend (San Diego, CA, USA). Peptide-MHC II tetramers were prepared and used as previously described ${ }^{16}$. Multi-color cytometry were performed on Canto II, LSRFortessa X-20 (both from BD Biosciences) and CyAn (Dako) flow cytometers, and analysed with FlowJo v10 (Tree Star, Ashland, OR, USA) or Summit v4.3 (Dako) analysis software.

Micropipette adhesion frequency assay. Two-dimensional TCR affinities were measured by a micropipette adhesion frequency assay as has been described in detail elsewhere ${ }^{24,25}$, using the T-cell hybridomas H5 and H18 and $\mathrm{A}^{\mathrm{b}}$-env $\mathrm{v}_{125-135}$ tetramer. Cells were brought into contact 50 times with the same contact time and area $\left(A_{\mathrm{c}}\right)$, and an adhesion frequency $\left(P_{\mathrm{a}}\right)$ was calculated. Surface pMHC and TCR $\beta$ densities were determined by flow cytometry and BD QuantiBRITE PE Beads for standardization (BD Biosciences). These parameters were then used to calculate two-dimensional affinity using the following equation: $A_{\mathrm{c}} \times K_{\mathrm{a}}=-\ln \left[1-P_{\mathrm{a}}(\infty)\right] m_{\mathrm{r}}^{-1} m_{\mathrm{l}}^{-1}$ where $m_{\mathrm{r}}$ and $m_{\mathrm{l}}$ represent TCR and pMHC surface densities, respectively.

In vitro T-cell activation. Spleen or lymph node single-cell suspensions were prepared from EF4.1 or EV $\alpha 3$ mice and $5 \times 10^{5}$ cells per well were stimulated in 96-well plates with the indicated amount of the index env ${ }_{122-141}$ epitope (DEPLTSLTPRCNTAWNRLKL), the shorter env ${ }_{124-138} \mathrm{~L}$ epitope (PLTSLTPRCNTAWNR) or the altered peptides epitopes env $v_{124-138} \mathrm{Y}$ (PLTSYTPRCNTAWNR) and env $124-138$ I (PLTSITPRCNTAWNR). Hybridoma cell lines were generated and stimulated as previously described ${ }^{23}$. T-cell activation was assessed $18 \mathrm{~h}$ later by flow cytometric detection of CD69 or CD44 (eBiosciences).

Next-generation sequencing of the TCR repertoire. For TCR sequencing, naive or primed env-specific EF4.1 CD4 ${ }^{+}$T-cell subsets were purified by cell sorting ( $>98 \%)$ and RNA was isolated using the QIAcube (QIAGEN, Crawley, UK). Synthesis of cDNA was carried out with the High Capacity Reverse Transcription kit (Applied Biosystems, Carlsbad, CA, USA) with an added RNase-inhibitor (Promega Biosciences, Madison, WI, USA). A final clean-up was performed with the QIAquick PCR purification kit (Qiagen). Purified cDNA was then used as template for the amplification of Trav14 (encoding V $\alpha 2$ )- or Trav9 (encoding V $\alpha 3$ )-containing rearrangements, using the following primers: Trav14 forward: 5'-CAAGCTTCAGTCTAGGAGGAATGGAC-3'; Trav 9 forward: 5'-CCAAGGCTCAGCCATGCTCCTGGC-3'; Trac common reverse: 5'-TAACTGGTACACAGCAGGTTCTGGG-3'. The forward Trav-specific primers were located in a promoter region common to all members of the respective Trav family and the common reverse was in the common constant region, thus amplifying the entire variable and joining coding region $(\sim 500 \mathrm{bp}$ product). Between 4,500 and 43,000 sequences per sample (450 bp median size) were obtained on a GS FLX System by GATC Biotech (Constance, Germany). Identification of productive rearrangements, Trav and Traj gene segment annotation and protein translation and CDR3 segment prediction were performed by the ImMunoGeneTics (IMGT) online tool HighV-QUEST (http://www.imgt.org) ${ }^{67}$.

Immunohistochemistry. Frozen OCT (Dako)-embedded spleen sections were fixed in cold acetone, stained with directly conjugated monoclonal antibodies (Supplementary Table 1) against B220 (AlexaFluor 647, BD Biosciences) and IgD (PE, eBiosciences). Sections were also stained for the T- and B-cell activation antigen Ly77 (clone GL7, FITC, BD Biosciences) and FITC fluorescence was amplified with the AlexaFluor 488 signal amplification kit (Molecular Probes), according to manufacturer's instructions. Stained sections were mounted in fluorescent mounting medium (Dako) and viewed with a Leica TCS SP2 AOBS confocal microscope. The images were acquired with Leica confocal software using the $20 \times$ or $40 \times$ objective lenses.

Statistical analyses. Statistical comparisons were made using SigmaPlot 12.0 (Systat Software, Germany). Parametric comparisons of normally distributed 
values that satisfied the variance criteria were made by unpaired Student's $t$-tests. Data that did not pass the variance test were compared with non-parametric two-tailed Mann-Whitney rank sum test. Comparison of $V \beta$ family expression, hierarchical clustering and heat-map production was with Qlucore Omics Explorer (Qlucore, Lund, Sweden).

\section{References}

1. Turner, S. J., Doherty, P. C., McCluskey, J. \& Rossjohn, J. Structural determinants of T-cell receptor bias in immunity. Nat. Rev. Immunol. 6, 883-894 (2006).

2. Stritesky, G. L., Jameson, S. C. \& Hogquist, K. A. Selection of self-reactive $\mathrm{T}$ cells in the thymus. Annu. Rev. Immunol. 30, 95-114 (2012).

3. Thorborn, G., Young, G. R. \& Kassiotis, G. Effective T helper cell responses against retroviruses: are all clonotypes equal? J. Leukoc. Biol. 96, 27-37 (2014).

4. Morris, G. P. \& Allen, P. M. How the TCR balances sensitivity and specificity for the recognition of self and pathogens. Nat. Immunol. 13, 121-128 (2012).

5. Gett, A. V., Sallusto, F., Lanzavecchia, A. \& Geginat, J. T cell fitness determined by signal strength. Nat. Immunol. 4, 355-360 (2003).

6. Fasso, M. et al. T cell receptor (TCR)-mediated repertoire selection and loss of TCR vbeta diversity during the initiation of a CD4 $(+) \mathrm{T}$ cell response in vivo. J. Exp. Med. 192, 1719-1730 (2000).

7. Malherbe, L., Hausl, C., Teyton, L. \& McHeyzer-Williams, M. G. Clonal selection of helper T cells is determined by an affinity threshold with no further skewing of TCR binding properties. Immunity 21, 669-679 (2004).

8. Ploquin, M. J., Eksmond, U. \& Kassiotis, G. B cells and TCR avidity determine distinct functions of CD4 + T cells in retroviral infection. J. Immunol. 187, 3321-3330 (2011).

9. Kim, C., Wilson, T., Fischer, K. F. \& Williams, M. A. Sustained interactions between $\mathrm{T}$ cell receptors and antigens promote the differentiation of CD4 $(+)$ memory T cells. Immunity 39, 508-520 (2013).

10. Weber, K. S. et al. Distinct CD4 + helper T cells involved in primary and secondary responses to infection. Proc. Natl Acad. Sci. USA 109, 9511-9516 (2012).

11. Persaud, S. P., Parker, C. R., Lo, W. L., Weber, K. S. \& Allen, P. M. Intrinsic $\mathrm{CD} 4(+) \mathrm{T}$ cell sensitivity and response to a pathogen are set and sustained by avidity for thymic and peripheral complexes of self peptide and MHC. Nat. Immunol. 15, 266-274 (2014).

12. Mandl, J. N., Monteiro, J. P., Vrisekoop, N. \& Germain, R. N. T cell-positive selection uses self-ligand binding strength to optimize repertoire recognition of foreign antigens. Immunity 38, 263-274 (2013).

13. Tubo, N. J. et al. Single naive CD4 $(+) \mathrm{T}$ cells from a diverse repertoire produce different effector cell types during infection. Cell 153, 785-796 (2013).

14. Malherbe, L., Mark, L., Fazilleau, N., McHeyzer-Williams, L. J. \& McHeyzerWilliams, M. G. Vaccine adjuvants alter TCR-based selection thresholds. Immunity 28, 698-709 (2008).

15. Baumgartner, C. K., Yagita, H. \& Malherbe, L. P. A TCR affinity threshold regulates memory $\mathrm{CD} 4 \mathrm{~T}$ cell differentiation following vaccination. J. Immunol. 189, 2309-2317 (2012).

16. Thorborn, G. et al. Clonotypic composition of the CD4+ T cell response to a vectored retroviral antigen is determined by its speed. J. Immunol. 193, 1567-1577 (2014)

17. Rees, W. et al. An inverse relationship between $\mathrm{T}$ cell receptor affinity and antigen dose during $\mathrm{CD} 4(+) \mathrm{T}$ cell responses in vivo and in vitro. Proc. Natl Acad. Sci. USA 96, 9781-9786 (1999).

18. Anderton, S. M., Radu, C. G., Lowrey, P. A., Ward, E. S. \& Wraith, D. C. Negative selection during the peripheral immune response to antigen. J. Exp. Med. 193, 1-11 (2001).

19. Hasenkrug, K. J. \& Chesebro, B. Immunity to retroviral infection: the Friend virus model. Proc. Natl Acad. Sci. USA 94, 7811-7816 (1997).

20. Tsuji-Kawahara, S. et al. Differential requirements of cellular and humoral immune responses for Fv2-associated resistance to erythroleukemia and for regulation of retrovirus-induced myeloid leukemia development. J. Virol. 87, 13760-13774 (2013).

21. Antunes, I. et al. Retrovirus-specificity of regulatory T cells is neither present nor required in preventing retrovirus-induced bone marrow immune pathology. Immunity 29, 782-794 (2008).

22. Kearney, E. R., Pape, K. A., Loh, D. Y. \& Jenkins, M. K. Visualization of peptide-specific $\mathrm{T}$ cell immunity and peripheral tolerance induction in vivo. Immunity 1, 327-339 (1994).

23. Young, G. R. et al. Negative selection by an endogenous retrovirus promotes a higher-avidity CD4 $+\mathrm{T}$ cell response to retroviral infection. PLoS Pathog. 8, e1002709 (2012).

24. Sabatino, Jr. J. J., Huang, J., Zhu, C. \& Evavold, B. D. High prevalence of low affinity peptide-MHC II tetramer-negative effectors during polyclonal CD4+ T cell responses. J. Exp. Med. 208, 81-90 (2011).

25. Huang, J. et al. The kinetics of two-dimensional TCR and pMHC interactions determine T-cell responsiveness. Nature 464, 932-936 (2010).
26. Kassiotis, G., Zamoyska, R. \& Stockinger, B. Involvement of avidity for major histocompatibility complex in homeostasis of naive and memory T cells. J. Exp. Med. 197, 1007-1016 (2003).

27. Martin, B. et al. Highly self-reactive naive CD4 T cells are prone to differentiate into regulatory T cells. Nat. Commun. 4, 2209 (2013).

28. Dittmer, U., Brooks, D. M. \& Hasenkrug, K. J. Characterization of a liveattenuated retroviral vaccine demonstrates protection via immune mechanisms. J. Virol. 72, 6554-6558 (1998).

29. Marques, R. et al. B lymphocyte activation by coinfection prevents immune control of Friend virus infection. J. Immunol. 181, 3432-3440 (2008).

30. Qi, H., Cannons, J. L., Klauschen, F., Schwartzberg, P. L. \& Germain, R. N. SAP-controlled T-B cell interactions underlie germinal centre formation. Nature 455, 764-769 (2008).

31. Marques, R. et al. Generalized immune activation as a direct result of activated CD4 + T cell killing. J. Biol. 8, 93 (2009).

32. Klarnet, J. P. et al. FBL-reactive CD8 + cytotoxic and CD4 + helper T lymphocytes recognize distinct Friend murine leukemia virus-encoded antigens. J. Exp. Med. 169, 457-467 (1989).

33. Smith, K. et al. Sensory adaptation in naive peripheral CD4 T cells. J. Exp. Med. 194, 1253-1261 (2001).

34. Kassiotis, G., Garcia, S., Simpson, E. \& Stockinger, B. Impairment of immunological memory in the absence of MHC despite survival of memory $\mathrm{T}$ cells. Nat. Immunol. 3, 244-250 (2002).

35. Moran, A. E. et al. T cell receptor signal strength in Treg and iNKT cell development demonstrated by a novel fluorescent reporter mouse. J. Exp. Med. 208, 1279-1289 (2011).

36. Hogquist, K. A. \& Jameson, S. C. The self-obsession of T cells: how TCR signaling thresholds affect fate 'decisions' and effector function. Nat. Immunol. 15, 815-823 (2014).

37. Fulton, R. B. et al. The TCR's sensitivity to self peptide-MHC dictates the ability of naive $\mathrm{CD} 8(+) \mathrm{T}$ cells to respond to foreign antigens. Nat. Immunol. 16, 107-117 (2015).

38. McHeyzer-Williams, M. G. \& Davis, M. M. Antigen-specific development of primary and memory T cells in vivo. Science 268, 106-111 (1995).

39. Savage, P. A., Boniface, J. J. \& Davis, M. M. A kinetic basis for T cell receptor repertoire selection during an immune response. Immunity 10, 485-492 (1999).

40. Kim, C., Jay, D. C. \& Williams, M. A. Stability and function of secondary Th1 memory cells are dependent on the nature of the secondary stimulus. $J$. Immunol. 189, 2348-2355 (2012).

41. Miconnet, I. et al. Large TCR diversity of virus-specific CD8 T cells provides the mechanistic basis for massive TCR renewal after antigen exposure. J. Immunol. 186, 7039-7049 (2011)

42. Buchholz, V. R. et al. Disparate individual fates compose robust CD8 + T cell immunity. Science 340, 630-635 (2013).

43. Gerlach, C. et al. Heterogeneous differentiation patterns of individual CD8 + T cells. Science 340, 635-639 (2013).

44. Ertelt, J. M. et al. Selective culling of high avidity antigen-specific CD4 + T cells after virulent Salmonella infection. Immunology 134, 487-497 (2011).

45. Caserta, S., Kleczkowska, J., Mondino, A. \& Zamoyska, R. Reduced functional avidity promotes central and effector memory CD4 $\mathrm{T}$ cell responses to tumorassociated antigens. J. Immunol. 185, 6545-6554 (2010).

46. McHeyzer-Williams, L. J., Panus, J. F., Mikszta, J. A. \& McHeyzer-Williams, M. G. Evolution of antigen-specific $\mathrm{T}$ cell receptors in vivo: preimmune and antigen-driven selection of preferred complementarity-determining region 3 (CDR3) motifs. J. Exp. Med. 189, 1823-1838 (1999).

47. Lund, F. E. \& Randall, T. D. Effector and regulatory B cells: modulators of CD4(+) T cell immunity. Nat. Rev. Immunol. 10, 236-247 (2010).

48. Dorner, T., Giesecke, C. \& Lipsky, P. E. Mechanisms of B cell autoimmunity in SLE. Arthritis Res. Ther. 13, 243 (2011).

49. Haas, A., Zimmermann, K. \& Oxenius, A. Antigen-dependent and independent mechanisms of T and B cell hyperactivation during chronic HIV-1 infection. J. Virol. 85, 12102-12113 (2011).

50. Moir, S. \& Fauci, A. S. B cells in HIV infection and disease. Nat. Rev. Immunol. 9, 235-245 (2009).

51. Turnbull, E. L. et al. Kinetics of expansion of epitope-specific T cell responses during primary HIV-1 infection. J. Immunol. 182, 7131-7145 (2009).

52. Vingert, B. et al. HIV controller CD4 + T cells respond to minimal amounts of Gag antigen due to high TCR avidity. PLoS Pathog. 6, e1000780 (2010).

53. Waskow, C. et al. The receptor tyrosine kinase Flt3 is required for dendritic cell development in peripheral lymphoid tissues. Nat. Immunol. 9, 676-683 (2008).

54. Serreze, D. V. et al. B lymphocytes are essential for the initiation of T cellmediated autoimmune diabetes: analysis of a new 'speed congenic' stock of NOD.Ig mu null mice. J. Exp. Med. 184, 2049-2053 (1996).

55. Vong, A. M. et al. Spectratyping analysis of the islet-reactive T cell repertoire in diabetic NOD Igmu(null) mice after polyclonal B cell reconstitution. J. Transl. Med. 9, 101 (2011).

56. Mamula, M. J. \& Janeway, Jr. C. A. Do B cells drive the diversification of immune responses? Immunol. Today 14, 151-152 (1993). 
57. Mombaerts, P. et al. RAG-1-deficient mice have no mature B and T lymphocytes. Cell 68, 869-877 (1992).

58. Kitamura, D., Roes, J., Kuhn, R. \& Rajewsky, K. A B cell-deficient mouse by targeted disruption of the membrane exon of the immunoglobulin mu chain gene. Nature 350, 423-426 (1991).

59. Wu, C. et al. SAP controls $\mathrm{T}$ cell responses to virus and terminal differentiation of TH2 cells. Nat. Immunol. 2, 410-414 (2001).

60. Philpott, K. L. et al. Lymphoid development in mice congenitally lacking T cell receptor alpha beta-expressing cells. Science 256, 1448-1452 (1992).

61. Cosgrove, D. et al. Mice lacking MHC class II molecules. Cell 66, 1051-1066 (1991).

62. Rothe, J. et al. Mice lacking the tumour necrosis factor receptor 1 are resistant to TNF-mediated toxicity but highly susceptible to infection by Listeria monocytogenes. Nature 364, 798-802 (1993).

63. Srinivas, S. et al. Cre reporter strains produced by targeted insertion of EYFP and ECFP into the ROSA26 locus. BMC. Dev. Biol. 1, 4 (2001).

64. Klinger, M. et al. Thymic OX40 expression discriminates cells undergoing strong responses to selection ligands. J. Immunol. 182, 4581-4589 (2009).

65. Kaji, T. et al. Distinct cellular pathways select germline-encoded and somatically mutated antibodies into immunological memory. J. Exp. Med. 209, 2079-2097 (2012).

66. Young, G. R. et al. Resurrection of endogenous retroviruses in antibodydeficient mice. Nature 491, 774-778 (2012).

67. Alamyar, E., Duroux, P., Lefranc, M. P. \& Giudicelli, V. IMGT(R) tools for the nucleotide analysis of immunoglobulin (IG) and T cell receptor (TR) V-(D)-J repertoires, polymorphisms, and IG mutations: IMGT/V-QUEST and IMGT/ HighV-QUEST for NGS. Methods Mol. Biol. 882, 569-604 (2012).

\section{Acknowledgements}

We wish to thank Dr Kristin A. Hogquist for the Nur77-GFP mice, Dr Toshitada Takemori for the conditional Bcl6 mice, Dr Kim Hasenkrug for FV and FBL-3 stocks, Dr Ulf Dittmer for the Ad5.pIX-gp70 stocks and Drs Anne O'Garra, Brigitta Stockinger, Mark Wilson and Eva Frickel for helpful comments and discussion. We are grateful for assistance from the Biological Services, Flow Cytometry and Protein Purification
Facilities at the Francis Crick institute. This work was supported by the Francis Crick Institute which receives its core funding from Cancer Research UK, the UK Medical Research Council (U117581330 to G.K.), and the Wellcome Trust.

\section{Author contributions}

J.M. performed and interpreted experiments and wrote the manuscript; M.J.P., U.E., G.T. and A.F. performed and interpreted experiments; R.A. and B.E. designed, performed and interpreted experiments relating to two-dimensional TCR affinity measurements. M.P. designed, prepared and provided the MHC II tetramer used in this study. G.K. designed and interpreted the experiments, wrote the manuscript and supervised the study.

\section{Additional information}

Accession codes: The Sequence Read Archive (SRA) and European Nucleotide Archive (ENA) accession numbers for the TCR sequences reported in this paper are SRX750575 and PRJEB11747, respectively.

Supplementary Information accompanies this paper at http://www.nature.com/ naturecommunications

Competing financial interests: The authors declare no competing financial interests.

Reprints and permission information is available online at http://npg.nature.com/ reprintsandpermissions/

How to cite this article: Merkenschlager, J. et al. Stepwise B-cell-dependent expansion of T helper clonotypes diversifies the T-cell response. Nat. Commun. 7:10281 doi: $10.1038 /$ ncomms10281 (2016).

(c) (i) This work is licensed under a Creative Commons Attribution 4.0 International License. The images or other third party material in this article are included in the article's Creative Commons license, unless indicated otherwise in the credit line; if the material is not included under the Creative Commons license, users will need to obtain permission from the license holder to reproduce the material. To view a copy of this license, visit http://creativecommons.org/licenses/by/4.0/ 